\title{
Technical Report: Research in Particle Physics at the Santa Cruz Institute for Particle Physics, 2000-2003.
}

\author{
Task A: Experimental Particle Physics
}

\section{GENERAL INTRODUCTION}

The Santa Cruz Institute for Particle Physics is an Organized Research Unit within the University of California system. This is a special structure allowing a focused emphasis on research and includes special commitments for space and personnel from the Santa Cruz campus. The Institute serves to consolidate the research in experimental and theoretical particle physics on campus. The Unit is separately administered and allows us to benefit from some of the best aspects of a laboratory, such as fully dedicated scientists and generous research space, and also some of the best aspects of a University, such as access to and interaction with graduate and undergraduate students. The Institute includes a DOE supported experimental and theoretical program (the focus of this technical report) as well as a separate NSF supported experimental group whose major focus is in ground-based Particle Astrophysics.

\section{THE EXPERIMENTAL PROGRAM}

\subsection{INTRODUCTION}

This report covers four separate experimental projects. The projects are the BaBar experiment, the ATLAS experiment, the GLAST space satellite, and work toward a Linear Collider and its detector.

Our institute has a long history of leadership activities in each of the four activities. In 1988 we were involved in the first detailed study of how one would do the CP violation measurement at an asymmetric B-factory, including the needed machine luminosity. This resulted in a four-author publication in the Physical Review, with two authors from SCIPP. We were then deeply involved in the organization of the BaBar experiment and the detector design. Our group made major contributions to the design of the front-end chip for the vertex detector, was responsible for the entire data transmission system for the vertex detector (led by A. Grillo, A. Seiden, and E. Spencer), designed and tested all the front-end chips for the drift chamber (D. Dorfan and B. Schumm), and led the overall computing effort for several years (T. Schalk). In the area of analysis SCIPP postdoctoral scientists led the effort to develop the tagging algorithm required for the $\mathrm{CP}$ measurement, co-lead the charm analysis working group, and have contributed to the analysis of the electromagnetic penguin decays.

Our work on ATLAS is based on major activities in silicon detectors starting first with the MARKII silicon vertex detector (the first at a colliding beam facility and the first using VLSI chips bonded directly to the silicon wafers, led at SCIPP by A. Litke). Building on this effort, much of the early work toward a very large silicon tracker was done in the context of the SDC detector for the SSC (led by D. Dorfan, A. Grillo, H. Sadrozinski and A. Seiden). This included a number of design features, now part of 
ATLAS, which were aimed at a cost-effective optimization of the detector tracking capabilities (silicon stereo detectors, the use of forward disks, the use of a binary readout architecture) as well as many key generic detector and electronics measurements (including type inversion in detectors; the potential for thermal runaway, and the use of TPG as a low-Z heat spreading material). Our group now provides the subsystem leader (A. Seiden) for the silicon construction activities of the U.S. ATLAS collaboration, which includes silicon strips, pixel detectors, and complex off-detector readout, calibration, and control interface boards. This effort involves seven universities and the Lawrence Berkeley National Laboratory. We also provide the leader of the entire ATLAS silicon strip electronics team (from front-ends to power supplies, led by A. Grillo), the silicon strip systems engineer (E. Spencer), and the leader of the ATLAS tracking display package, called ATLANTIS (A. Litke).

SCIPP has played a key role in the evolution and development of the GLAST project almost from its conception by Bill Atwood, then at SLAC. There are many challenges in realizing a large silicon strip detector suitable for operation in space, and key among them is the development of low-power electronics for the readout system. The nascent GLAST collaboration eagerly sought the participation of SCIPP because of their long history and success in building silicon-strip detector systems. Robert Johnson took up this challenge in 1995 and by the end of 1999 had demonstrated a complete Tracker module with $>50,000$ channels that met the stringent GLAST power and noise requirements. Johnson is now the subsystem manager for the GLAST Tracker, comprised of over about $80 \mathrm{~m}^{2}$ of silicon-strip detectors and almost a million readout channels. This hardware effort is presently moving from design and prototyping into fabrication of flight hardware, with SCIPP responsible for delivery of the electronic readout system as well as management of the overall effort. Hartmut Sadrozinski early on organized the design and procurement of the silicon detectors. His expertise and long experience in this technology led to a very successful collaboration with Hiroshima University in Japan to purchase more than 11,000 detectors. Sadrozinski is the GLAST Tracker Scientist and has been leading efforts to procure and space-qualify other key Tracker components in addition to the detectors. During the same period Bill Atwood, on sabbatical at UCSC from SLAC, developed most of the simulation code and analysis that validated the GLAST design, demonstrating that more than a factor of 50 improvement in sensitivity relative to the previous gamma-ray mission was at hand. Post Docs and students working with Atwood and Johnson took this first analysis package and developed the second-generation code used for the final proposal, which was accepted by both DOE and NASA in 2000. Atwood, now a Principal Investigator at SCIPP, recently completed a third re-write of the code, incorporating new techniques to closely couple the simulation to the analysis and implement new (to HEP) statistical methods that dramatically improve the efficiency of event selection and filtering.

SCIPP physicists have been very active on Linear Collider development for the last few years. Areas of interest include the physics and machine requirements for an $\mathrm{e}^{-} \mathrm{e}^{-}$Linear Collider option, research and development towards the optimization of the central tracker for a Linear Collider detector, and physics studies of QCD, supersymmetry, and alternative symmetry-breaking signals that might be observed at a high energy $\mathrm{e}^{+} \mathrm{e}^{-}$ colliding beam facility. The international effort toward an $\mathrm{e}^{-} \mathrm{e}^{-}$machine has been led by 
C.A. Heusch. Heusch has organized four workshops on the Santa Cruz campus whose proceedings provide much of the community's reference material on the $\mathrm{e}^{-} \mathrm{e}^{-}$option. $\mathrm{B}$. Schumm is playing a major role in the development and optimization of tracking systems for the Linear Collider detector. He is currently the co-leader of the tracking subgroup of the American Linear Collider Physics Group, the umbrella organization of North American university and laboratory research groups interested in Linear Collider development work. Before taking on his current role as tracking group co-convener, Schumm acted as co-convener and international contact for the Linear Collider QCD physics study group. 


\subsection{BABAR DETECTOR AT THE PEP-II B FACTORY}

\subsubsection{Introduction}

The BaBar Detector is currently taking data at IR-2, the sole instrumented interaction region of the PEP-II storage ring at SLAC. The PEP-II machine is designed to run with asymmetric beam energies (9 $\mathrm{GeV}$ electrons and $3.1 \mathrm{GeV}$ positrons) at the $\mathrm{Y}(4 \mathrm{~S})$ resonance, yielding $\mathrm{B}$ meson pairs with a boost of $\beta \gamma=0.56$ in the frame of the BaBar Detector. With an initial design luminosity of $3 \times 10^{33} \mathrm{~cm}^{-2} \mathrm{~s}^{-1}$, the PEP-II facility has achieved a record luminosity of $4.6 \times 10^{33} \mathrm{~cm}^{-2} \mathrm{~s}^{-1}$, and is an optimal venue for the study of $\mathrm{CP}$ violation in the $\mathrm{B}$ meson system.

In the thirty years that have elapsed since the surprising discovery of CP violation in $K_{L}^{0}$ meson decay, our understanding of elementary particle physics has evolved considerably. The Standard Model with three quark generations accommodates CP violation in quark decays in an elegant and economical manner. Through one imaginary parameter and three real numbers, the Standard Model completely characterizes the weak couplings of the six quarks as expressed in the Cabibbo-Kobayashi-Maskawa (CKM) matrix. We have had little evidence, however, that this explanation is correct. Indeed, there are hints from cosmological arguments that it is not sufficient: attempts to understand the observed baryon/antibaryon asymmetry in the universe in the context of the Standard model have so far proved unsuccessful. Thus, any interpretable measurement of CP-violating effects outside the Kaon system are of fundamental interest to the fields of both particle physics and cosmology.

The B meson system is an ideal window into CP physics. Since the B meson contains a third-generation quark, the participation of all three generations in B meson decay can be relatively democratic. In such cases, the relative imaginary phase of the CKM matrix elements will not be hidden in highly suppressed contributions to the decay process, and CP-violating effects can be large. It is a primary goal of the PEP-II/BaBar facility to make precise, interpretable measurements of CP-violating effects on the B meson system, thereby probing the sufficiency of the Standard Model as the source of CP violation, and possibly providing a window into physics beyond the Standard Model.

The BaBar Detector at PEP-II began taking physics data in 1999. Its first substantial run (RUN I), from January through October 2000, integrated a sample of $20.2 \mathrm{fb}^{-1}$ on the Y (4S) peak. The second completed run of PEP-II took place between January 2001 and June 2002, integrating an additional sample of $61.2 \mathrm{fb}^{-1}$ of on-peak data. RUN-III began in November 2002, and is expected to extend to August 2003, with an expectation of accumulating an additional $50 \mathrm{fb}^{-1}$ of on-peak data. This run profile is summarized in Figure 2.2-1. In addition to the on-peak data, BaBar/PEP-II has integrated another 12\% of data at a center-of-mass energy of $10.540 \mathrm{GeV}, 40 \mathrm{MeV}$ below the Y (4S) peak. 
Table 2.2-1. BaBar data samples.

\begin{tabular}{|l|l|l||}
\hline RUN & DURATION & ON-PEAK SAMPLE (GOAL) \\
\hline RUN I & $01 / 00-10 / 00$ & $20 \mathrm{fb}^{-1}$ \\
\hline RUN II & $01 / 01-06 / 02$ & $61 \mathrm{fb}^{-1}$ \\
\hline RUN III & $11 / 02-08 / 03$ & $\left(50 \mathrm{fb}^{-1}\right)$ \\
\hline
\end{tabular}

The BaBar project has been the largest effort within SCIPP over the past few years. Active participants in the BaBar project include three faculty, one adjunct faculty, one senior research scientist, three postdoctoral scientists and four graduate students. Two post-doctoral fellows (Juerg Beringer and Wolfgang Walkowiak; the latter half-time on Linear Collider R\&D) have recently left for career positions at CERN and the University of Siegen, respectively. An offer to replace Walkowiak's half-time BaBar/ half-time Linear Collider position has been accepted by Gavin Nesom, who is finishing his Ph.D. at Oxford and will begin working at SCIPP in May 2003. An offer to replace Beringer's full-time BaBar position has been accepted by Juergen Kroseberg, who has recently finished his Ph.D. at the University of Zurich, and who will begin working at SCIPP in June, 2003.

The physics program of BaBar is broad, entailing much more than just the flagship CP violation studies that initiated the PEP-II/BaBar program. SCIPP physicists and students have, in recent years, made critical contributions to three of the most visible research arenas within BaBar: $\mathrm{CP}$ violation studies, the study of meson/antimeson mixing in heavy quark systems, and the study of radiative penguin decays. The background and status of these areas of study are presented in the following three sections.

\subsubsection{Physics with BaBar}

\subsubsection{CP Physics with BaBar}

The Standard Model predictions for CP violation are most often presented in terms of the unitarity constraints for the three-generation CKM matrix. Unitarity of the CKM matrix requires

$$
V_{t b} V_{t d}^{*}+V_{c b} V_{c d}^{*}+V_{u b} V_{u d}^{*}=0
$$

The three complex quantities $\mathrm{V}_{\mathrm{id}} \mathrm{V}_{\mathrm{ib}}{ }^{*}$ can be represented as a triangle in the complex plane. The three angles of this triangle are labeled

$$
\alpha \equiv \arg \left(-\frac{V_{t d} V_{t b}^{*}}{V_{u d} V_{u b}^{*}}\right)
$$




$$
\begin{gathered}
\beta \equiv \arg \left(-\frac{V_{c d} V_{c b}^{*}}{V_{t d} V_{t b}^{*}}\right) \\
\gamma \equiv \arg \left(-\frac{V_{u d} V_{u b}^{*}}{V_{c d} V_{c b}^{*}}\right)
\end{gathered}
$$

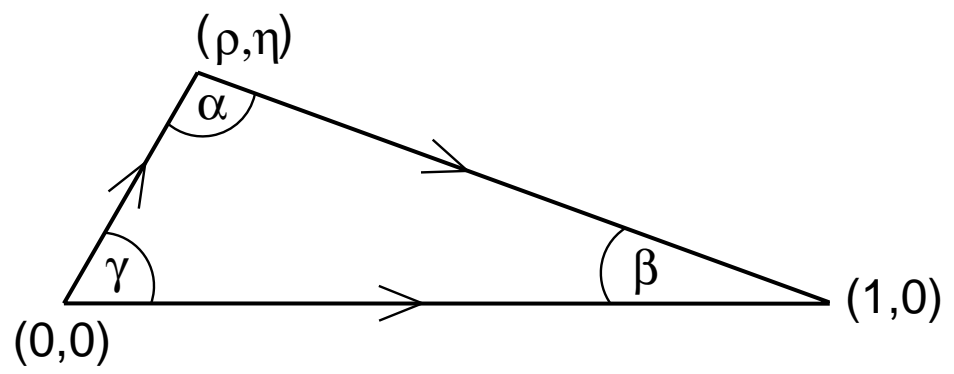

Figure 2.2-1: The Unitarity Triangle

Figure 2.2-1 shows the unitarity triangle, as it is usually drawn, rescaled by the side $\mathrm{V}_{\mathrm{cd}} \mathrm{V}_{\mathrm{cb}}{ }^{*}$ so that the base of the triangle is real and of unit length. The apex of the triangle is then the point $(\rho, \eta)$ in the complex plane, as first introduced by Wolfenstein [1].

The angles of the unitarity triangle can be directly measured via time-dependent $\mathrm{CP}$ asymmetries in the decay of $\mathrm{B}^{0}$ mesons into CP-eigenstate $f$ :

$$
a_{C P}^{f}(t)=\frac{\Gamma\left(B^{0}(t) \rightarrow f\right)-\Gamma\left(\bar{B}^{0}(t) \rightarrow f\right)}{\Gamma\left(B^{0}(t) \rightarrow f\right)+\Gamma\left(\bar{B}^{0}(t) \rightarrow f\right)}
$$

This CP asymmetry is related to combinations of CKM matrix elements according to

$$
a_{C P}^{f}(t)=\frac{\left(1-\left|r_{C P}(f)\right|^{2}\right) \cos (\Delta M t) \mp 2 \operatorname{Im} r_{C P}(f) \sin \Delta M t}{1+\left|r_{C P}(f)\right|^{2}},
$$

where $\Delta \mathrm{M}$ is the mass difference between the heavy and light $\mathrm{B}^{0}$ mass eigenstates, and the “-” (“+”) sign applies to CP even (odd) final states. The decay-mode dependent CPviolation factor $\mathrm{r}_{\mathrm{CP}}(f)$ is given by

$$
r_{C P}(f)=\frac{\bar{A}}{A} e^{2 i \phi_{M}}
$$

where 


$$
e^{2 i \phi_{M}}=\frac{V_{t b}^{*} V_{t d}}{V_{t b} V_{t d}^{*}}
$$

is the $B^{0}-\bar{B}^{0}$ mixing phase, and

$$
A=\left\langle f|\mathrm{H}| B^{0}\right\rangle \quad \bar{A}=\left\langle f|\mathrm{H}| \bar{B}^{0}\right\rangle
$$

are the respective matter and antimatter decay amplitudes. When only a single amplitude with a given weak decay phase $\phi_{\mathrm{D}}$ dominates the decay, one has

$$
\frac{\bar{A}}{A}=e^{-2 i \phi_{D}} .
$$

In this case, $\left|r_{C P}(f)\right|=1$ and $\operatorname{Im} r_{C P}(f)=\sin 2\left(\phi_{M}-\phi_{D}\right)$, and so the asymmetry then simplifies to

$$
a_{f}(t)=-2 \sin 2\left(\phi_{M}-\phi_{D}\right) \sin \Delta M t
$$

Table 2.2-2 shows the Standard Model relationship between the angles of the unitarity triangle and the predicted $\arg r_{C P}(f)=2\left(\phi_{M}-\phi_{D}\right)$, assuming a single weak decay amplitude dominates in each of these cases. In addition to pure CP eigenstates, other channels with CP-self-conjugate quark content can be analyzed for CP-violating contributions. These include mixed spin states such as $J / \psi K^{*}$ and vector-scalar mixtures such as $\rho \pi$ and $D^{*} D$ where the various possible charge assignments have both $\mathrm{CP}$ and isospin relationships [2][3]. 
Table 2.2-2: CP modes and Standard Model asymmetry predictions for $\mathrm{B}_{\mathrm{d}}$ modes.

\begin{tabular}{||l|l|l||}
\hline Quark Process & B $_{\mathbf{d}}$ Mode & $\phi_{M}-\phi_{D}$ \\
\hline$b \rightarrow c \bar{c} S$ & $J / \psi K_{S}^{0}$ & $\beta$ \\
& $J / \psi K_{L}^{0}$ & \\
& $J / \psi K^{0^{*}}$ & \\
\hline$b \rightarrow c \bar{c} d$ & $D^{+} D^{-}$ & $\beta$ \\
& $D^{*+} D^{*_{-}}$ & \\
& $D^{ \pm *} D^{\mp}$ & \\
\hline$b \rightarrow u \bar{u} d, d \bar{d} d$ & $\pi^{+} \pi^{-}$ & $\alpha$ \\
& $\rho^{ \pm} \pi^{\mp}$ & \\
& $a_{1}^{ \pm} \pi^{\mp}$ & \\
\hline
\end{tabular}

With an $\mathrm{e}^{+} \mathrm{e}^{-} \mathrm{B}$ Factory, the initial $B^{0}$ and $\bar{B}^{0}$ are produced in a coherent $B^{0} \bar{B}^{0}$ state and remain in this state until one of the particles decays. If one $B$ decays to a mode which establishes its flavor, while the other decays to a CP-study mode, the event can be used to reconstruct the time dependence of the asymmetry. In this case, the time $t$ in the equations above is the time between the tagging decay and the CP-study-mode decay. The tagging decay may be the later decay, in which case $t<0$. Thus, the time-integrated CP asymmetry vanishes if $\left|r_{C P}(f)\right|=1$, and so the determination of the time evolution of the CP asymmetry $a_{C P}^{f}(t)$ is an essential component of CKM parameter measurements. This determination is only possible due to the asymmetric machine configuration and the precise vertex reconstruction capabilities of the BaBar detector. In addition, it should be noted that pure and efficient "tagging" schemes, i.e., algorithms that determine the flavor of the non-CP decay, are also an essential ingredient of the CKM parameter measurement. The BaBar group at SCIPP has played an essential role in the development and operation of the Silicon Vertex Tracker (SVT) as well as the development and implementation of tagging algorithms, as will be described below.

Based on the combined RUN-I and RUN-II data samples, BaBar has measured [4]

$$
\sin 2 \beta=0.741 \pm 0.067 \pm 0.034
$$

compared to the BELLE measurement [5] of

$$
\sin 2 \beta=0.719 \pm 0.074 \pm 0.035
$$




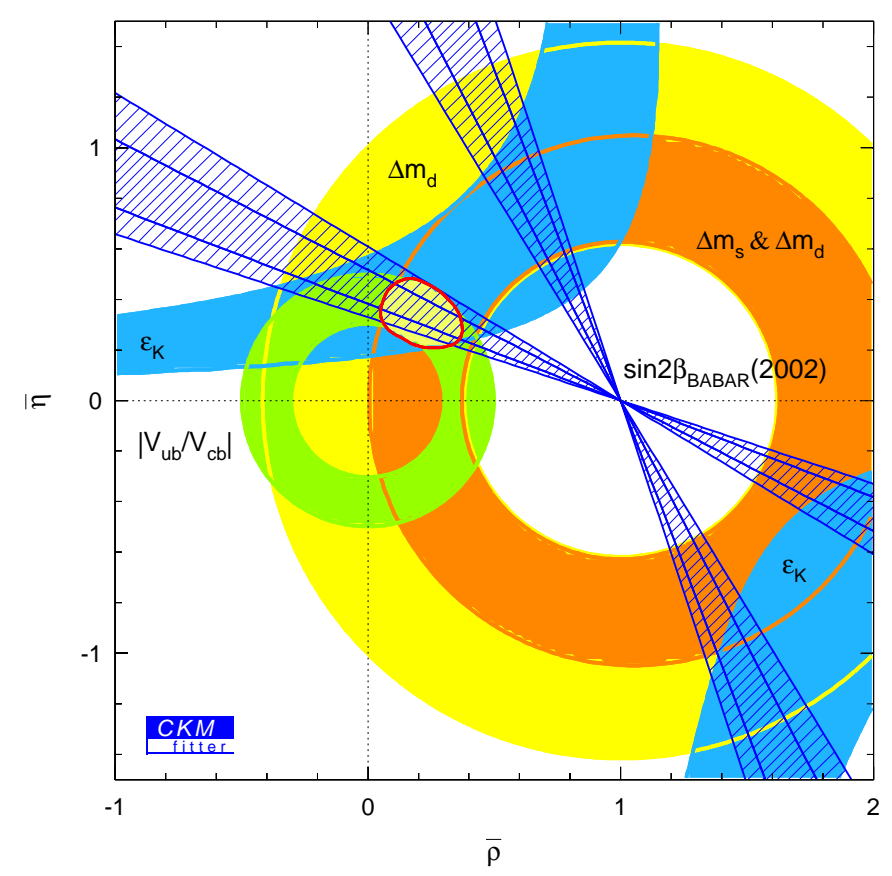

Figure 2.2-2: Current constraints on the unitarity triangle. The constraint from $\sin 2 \beta$ is that from BaBar alone, showing the $68 \%$ and $90 \%$ CL zones allowed by the BaBar result.

The oval in the lower part of the upper-right quadrant is the area allowed by all constraints other than those provided by $\sin 2 \beta$ measurements.

from the KEK-B factory in Japan. Constraints on the CKM unitarity triangle are shown in Figure 2.2-2, including the BaBar constraint on $\sin 2 \beta$. While all measurements are mutually consistent with the Standard-Model interpretation, the precision to which the unitarity triangle is constrained is markedly better than that of pre-B-factory running.

\subsubsection{Heavy-Flavor Mixing with BaBar}

The existence of higher-order diagrams that cause transitions between conjugate neutral meson states admits the possibility that the weak decay eigenstates are formed from a mixture of flavor eigenstates. For a heavy-quark system $\mathrm{H}$, we write for the mass eigenstates $\mathrm{H}_{1}$ and $\mathrm{H}_{2}$

$$
\begin{aligned}
& \left|H_{1}\right\rangle=p\left|H^{0}\right\rangle+q\left|\bar{H}^{0}\right\rangle \\
& \left|H_{2}\right\rangle=p\left|H^{0}\right\rangle-q\left|\bar{H}^{0}\right\rangle
\end{aligned}
$$

where $H^{0}, \bar{H}^{0}$ are the flavor eigenstates, and $|p|^{2}+|q|^{2}=1$. 
This mixing leads, in general, to differences in the $\mathrm{H}_{1}$ and $\mathrm{H}_{2}$ masses and lifetimes. We define mixing parameters $x$ and $y$ according to

$$
\begin{gathered}
x=2 \frac{M_{2}-M_{1}}{\Gamma_{1}+\Gamma_{2}} \equiv \frac{\Delta M}{\Gamma} \\
y=\frac{\Gamma_{1}-\Gamma_{2}}{\Gamma_{1}+\Gamma_{2}} \equiv \frac{\Delta \Gamma}{2 \Gamma} .
\end{gathered}
$$

In the $\mathrm{B}^{0}$ system, near-maximal coupling to the top quark leads to a large mass difference between weak eigenstates, and a correspondingly large $B^{0} \bar{B}^{0}$ transition rate. The current world average of [6]

$$
\Delta M=(0.489 \pm 0.008) p s^{-1}
$$

is derived from a broad spectrum of experimental results from hadron colliders and lepton colliders running both at the $\mathrm{Y}(4 \mathrm{~S})$ and $\mathrm{Z}^{0}$ pole, and corresponds to a timeintegrated mixing probability of $(18.1 \pm 0.4) \%$. Currently, use of this measurement to constrain the length of the $\mathrm{V}_{\mathrm{td}} \mathrm{CKM}$ element is limited by the understanding of longdistance effects in the hadronic matrix element; observation and quantification of $\mathrm{B}_{\mathrm{s}}$ mixing would allow these uncertainties to be normalized away, leading to an essential unitarity triangle constraint.

For the charm $\left(D^{0}\right)$ system, the CKM-suppression of the coupling to the third generation leads to much smaller mixing rates. The Standard Model expectation for $x$ and $y$ are both small -- of order $10^{-3}$ - and are dominated by radiative corrections, final-state interactions, and phase-space effects [7]. As such, the search for mixing effects in the $\mathrm{D}^{0}$ system holds substantial promise for the discovery of new physics [8][9].

At these low rates, doubly-Cabibbo-suppressed decay (DCSD) modes provide irreducible contributions to the time-integrated mixing rate. In terms of the ratio $R_{D}$ of DCSD to Cabibbo-favored decays, and in the limit of CP conservation in the $\mathrm{D}^{0}$ system, the time evolution of the mixing signal can be written as

$$
\Gamma\left(D^{0} \rightarrow K^{+} \pi^{-}\right)(t) \propto e^{-t / \tau_{D^{0}}}\left(R_{D}+\sqrt{R_{D}} y^{\prime} t / \tau_{D^{0}}+\frac{x^{\prime 2}+y^{\prime 2}}{4}\left(t / \tau_{D^{0}}\right)^{2}\right),
$$

allowing in principle for the separation of the DCSD and oscillation contributions. Here,

$$
\begin{gathered}
x^{\prime}=x \cos \delta+y \sin \delta \\
y^{\prime}=-x \sin \delta+y \cos \delta
\end{gathered}
$$


where $\delta$ is a decay-mode dependent strong phase that is essentially a free parameter in the mixing analysis.

It is also possible to constrain $x$ and $y$ by measuring the decay-time distribution of $\mathrm{D}^{0}$ decays to specific final states. For example, the decay $D^{0} \rightarrow K^{+} K^{-}$is to a CP-even state that is due, in the limit of CP conservation, purely to $\mathrm{D}_{1}$ decays. The lifetime $\tau_{K K}$ of such a sample is thus just $\tau_{K K}=1 / \Gamma_{1}$. The Cabibbo-favored decay to the CP-mixed state $D^{0} \rightarrow K^{-} \pi^{+}$is from an equal mixture of $\mathrm{D}_{1}$ and $\mathrm{D}_{2}$ states with the decay-time distribution

$$
N(t) \propto e^{-\Gamma t}\left(1+y^{\prime} \sqrt{R_{D}} \Gamma t+\frac{1}{2}\left(y^{2}-x^{2}\right) \Gamma^{2} t^{2}\right) .
$$

For small values of $x$ and $y$, this reduces to a simple exponential with a lifetime $\tau_{K \pi}$ that is the inverse of the average width $\Gamma$. In this limit, $y$ is measured directly from the ratio of the $K K$ and $K \pi$ lifetimes:

$$
y \cong \frac{\tau_{K \pi}}{\tau_{K K}}-1
$$

The analyses of the BaBar charm lifetime and mixing group, whose efforts are led by SCIPP post-doctoral fellow David Williams, provide the most accurate results currently available from any experiment worldwide. The lifetime-difference analysis, based on the full $91 \mathrm{fb}^{-1}$ sample of $K^{-} \pi^{+}, K^{-} K^{+}$, and $\pi^{-} \pi^{+}$decays, yields the result [10]

$$
y=0.0076_{-0.0057}^{+0.0061}(\text { stat. } \oplus \text { syst. })
$$

over twice as precise as any previous measurement [11][12][13][14]. The $D^{0} \rightarrow K^{+} \pi^{-}$ mixing analysis, based on the $57 \mathrm{fb}^{-1}$ of data taken through calendar year 2001, yields a measurement of the wrong-sign decay rate of [15]

$$
R_{W S}=[0.357 \pm 0.022(\text { stat. }) \pm 0.027(\text { syst. })] \%
$$

from which 95\% confidence limits

$$
\begin{gathered}
\left(x^{\prime}\right)^{2}<0.0020 \\
-0.027<y^{\prime}<0.022
\end{gathered}
$$

are derived. Prior measurements [16][17] are dominated by CLEO's measurement of $R_{W S}=(0.332 \pm 0.064 \pm 0.040) \%$, from which they derive $95 \%$ confidence limits of 
$\left(x^{\prime}\right)^{2}<0.0004$ and $-0.058<y^{\prime}<0.010$. Again, BaBar has provided a leading set of results in a critical arena.

\subsubsection{Radiative Penguin Decays with BaBar}

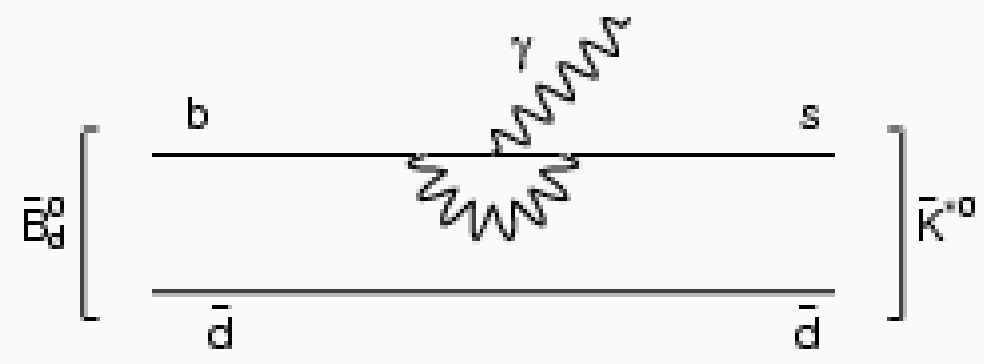

Figure 2.2-3: Example of a one-loop effective flavor-changing neutral current ('penguin') process.

The $b \rightarrow s \gamma$ transition, as an effective neutral current process, is suppressed within the Standard Model, taking place via the higher order "radiative penguin" process shown in Figure 2.2-3. Examination of this diagram suggests that the $b \rightarrow s \gamma$ transition rate is sensitive to new physics scenarios that introduce additional weakly-coupled bosons, such as the additional Higgs states of Supersymmetry. 


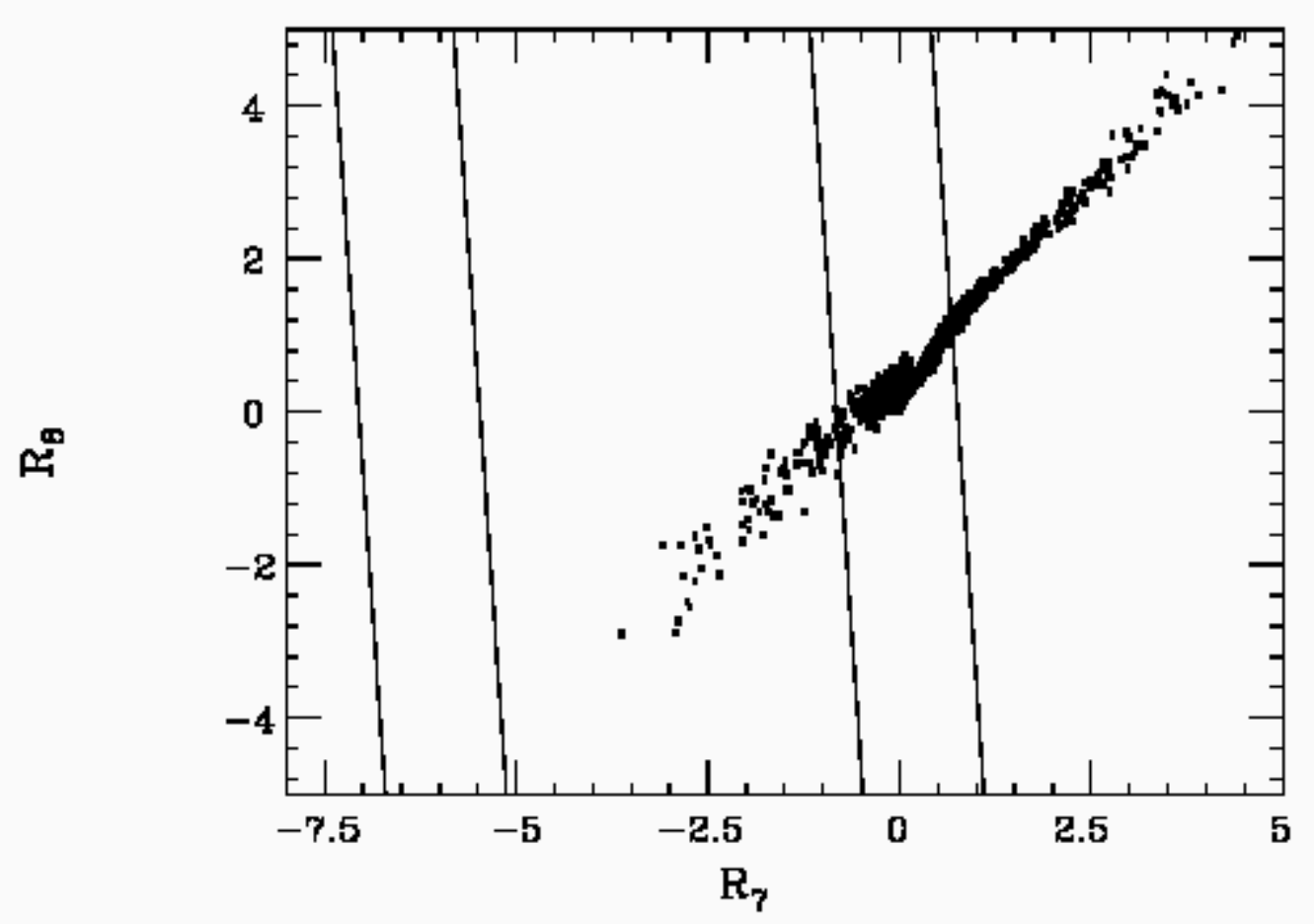

Figure 2.2-4: Typical constraints (68\% and 90\% CL) from radiative penguin decays on minimal supergravity models.

In the language of the Operator Product Expansion, the hadron-level realization $B \rightarrow X_{s} \gamma$ of the $b \rightarrow s \gamma$ transition is induced at leading order by the magnetic dipole $\left(\mathrm{O}_{7}\right)$ and chromomagnetic dipole $\left(\mathrm{O}_{8}\right)$ operators. Measurements of the $B \rightarrow X_{s} \gamma$ rate thus place constraints on the two-dimensional space of the corresponding operator strengths, or Wilson coefficients, $\mathrm{R}_{7}$ and $\mathrm{R}_{8}$. Figure $2.2-4$ shows the $68 \%$ and $90 \%$ confidence-level constraints provided by a typical measurement of the $B \rightarrow X_{s} \gamma$ rate - that of the CLEO collaboration [19] - of

$$
B\left(B \rightarrow X_{s} \gamma\right)=\left[3.21 \pm 0.43(\text { stat. }) \pm 0.27(\text { syst. })_{-0.10}^{+0.18}(\text { theo. })\right] \times 10^{-4}
$$

Also shown are points representative of the phase space of possible minimal supergravity (SUGRA) models, in view of constraints provided by direct searches and precision $\mathrm{Z}^{0}$ pole running. The measurement of the $b \rightarrow s \gamma$ rate is seen to provide a substantial constraint on the SUGRA model space, indicating its value as a probe of new physics.

SCIPP physicists have played a major role in BaBar's measurement of the $B \rightarrow X_{s} \gamma$ transition rate. This rate is measured via two independent approaches: a fully-inclusive measurement based on the inclusive spectrum of photons between 2.1 and $2.7 \mathrm{GeV}$, and a 'semi-inclusive' approach based on the full reconstruction of 12 exclusive modes that 
together account for approximately $50 \%$ of the $X_{s} \gamma$ decay width. Applying the inclusive approach to the $57 \mathrm{fb}^{-1}$ of data accumulated through calendar year 2001, BaBar has found [20]

$$
B\left(B \rightarrow X_{s} \gamma\right)=\left[3.38 \pm 0.36(\text { stat. }) \pm 0.37(\text { syst. })_{-0.28}^{+0.43}(\text { theo. })\right] \times 10^{-4}
$$

while on $20.7 \mathrm{fb}^{-1}$ of RUN-I data, the semi-inclusive approach has yielded [21]

$$
B\left(B \rightarrow X_{s} \gamma\right)=[4.2 \pm 0.5(\text { stat. }) \pm 0.8(\text { syst. }) \pm 1.3(\text { theo. })] \times 10^{-4}
$$

In addition to the CLEO result cited above, the $B \rightarrow X_{s} \gamma$ rate has been measured by BELLE [22]

$$
B\left(B \rightarrow X_{s} \gamma\right)=[3.36 \pm 0.53(\text { stat. }) \pm 0.42(\text { syst. }) \pm 0.52(\text { theo. })] \times 10^{-4}
$$

as well as at the $\mathrm{Z}^{0}$-pole by ALEPH [23]

$$
B\left(B \rightarrow X_{s} \gamma\right)=[3.11 \pm 0.80(\text { stat. }) \pm 0.72(\text { syst. })] \times 10^{-4}
$$

Again, BaBar is providing a leading measurement of an essential B-physics observable.

In addition to experimental uncertainty, use of the measured rate of $b \rightarrow s \gamma$ to constrain proposed models of new physics is limited by uncertainty on the theoretical prediction of the transition rate. Within the Standard Model,

$$
B\left(B \rightarrow X_{s} \gamma\right)=\frac{\left|V_{t s}^{*} V_{t b}\right|^{2}}{\left|V_{c b}\right|^{2}} \frac{6 \alpha}{\pi f(z)}\left|R_{7}^{(0) e f f}(\mu)\right|^{2} B\left(B \rightarrow X_{c} e \bar{v}_{e}\right)
$$

where $f\left(z=m_{c} / m_{b}\right)$ is the decay phase-space factor, and $\mu$ the QCD renormalization scale. A calculation at next-to-leading order by Gambino and Misiak [24] yields the Standard Model prediction $B(B \rightarrow s \gamma)=(3.60 \pm 0.30) \times 10^{-4}$; a calculation underway at next-to-next-to-leading order is expected to substantially reduce the uncertainty on this prediction.

\subsubsection{BaBar References}

[1] L. Wolfenstein, Phys. Rev. D31, (1985) 2381. 
[2] R. Aleksan, I. Dunietz, B. Kayser, and F. LeDiberder, Nucl. Phys. B361, (1991) 161.

[3] R. Aleksan, L.Oliver, O. Pene, and J.C. Raynal, Phys. Lett. B317, (1993) 123.

[4] B. Aubert et al. (BaBar Collaboration), Phys. Rev. Lett. 89, (2002), 281802.

[5] K. Abe et al. (BELLE Collaboration), Phys. Rev. D66, (2002) 071102.

[6] K. Hagiwara et al. (Particle Data Group), ' $B^{0}-\bar{B}^{0} \quad$ Mixing', http://pdg.lbl.gov/2002/bbmix_s042224.pdf (2002).

[7] F. Buccella, M. Lusignoli, and A. Pugliese, Phys. Lett. B379 (1996) 249.

[8] Ikaros I.Y. Bigi, ' $D^{0}-\bar{D}^{0}$ Mixing and CP Violation in $D$ Decays: Can There Be High-Impact Physics in Charm Decays?' Proceedings, Stanford Tau-Charm Factory Workshop, 1989:0169-195 (QCD183:T3:1989).

[9] H. Nelson, Compilation of $D^{0}-\bar{D}^{0}$ Mixing Prediction, Proceedings of the $19^{\text {th }}$ International Symposium on Lepton-Photon Interactions, Stanford, CA, August 9-14, 1999, hep-ex/9908021.

[10] B. Meadows (for the BaBar Collaboration), 'Charm Physics in Babar', to appear in the proceedings of the $38^{\text {th }}$ Rencontres de Moriond, March 23-29, 2003.

[11] K. Abe et al (Belle Collaboration), Phys. Rev. Lett. 88 (2002) 162001.

[12] G. Bonvicini et al. (CLEO Collaboration), Phys. Rev. Lett. 82 (1999) 4586.

[13] E. M. Aitala et al. (E791 Collaboration), Phys. Rev. Lett. 83 (1999) 32.

[14] J. M. Link et al. (FOCUS Collaboration), Phys. Lett. B485 (2000) 62.

[15] B. Aubert et al. (BaBar Collaboration), 'Search for $D^{0}-\bar{D}^{0}$ Mixing and a Measurement of the Doubly Cabibbo-suppressed Decay Rate in $D^{0} \rightarrow K \pi$ Decays', hep-ex/0304007, April, 2003 (submitted to Physical Review Letters).

[16] R. Godang et al. (CLEO Collaboration), Phys. Rev. Lett. 84 (2000) 5038.

[17] E. M. Aitala et al. (E791 Collaboration), Phys. Rev. D57 (1998) 13.

[18] G. Eigen et al., 'Indirect Investigations of Supersymmetry', Proceedings of the Snowmass 2001 Workshop on the Future of Particle Physics, Paper P342, Snowmass, Colorado, June 30-July 21, 2001.

[19] S. Chen et al. (CLEO Collaboration), Phys. Rev. Lett. 87, (2001) 251807. 
[20] B. Aubert et al. (BaBar Collaboration), 'Determination of the Branching Fraction for Inclusive Decays $B \rightarrow X_{s} \gamma$ ', to appear in the proceedings of the $31^{\text {st }}$ International Conference on High Energy Physics, Amsterdam, NL, July 24-31, 2002.

[21] B. Aubert et al. (BaBar Collaboration), ' $b \rightarrow s \gamma$ Using a Sum of Exclusive Modes', Proceedings, 31 ${ }^{\text {st }}$ International Conference on High Energy Physics, Amsterdam, The Netherlands, July 24-31, 2002, hep-ex/0207074.

[22] K. Abe et al. (BELLE Collaboration), Phys. Lett. B511 (2001) 151.

[23] R. Barate et al. (ALEPH Collaboration), Phys. Lett. B429 (1998) 169.

[24] Paolo Gambino and Mikolaj Misiak, Nucl.Phys. B611 (2001) 338.

[25] B. A. Schumm, `DE/DX Particle Identification with a Five Layer Silicon Tracker', SLAC-BABAR-NOTE-126, February, 1994.

[26] B. Aubert et al. (Babar Collaboration), Phys. Rev. Lett. 87 (2001), 091801.

[27] B. Aubert et al. (Babar Collaboration), Phys. Rev. Lett. 87 (2001), 241803.

[28] C. Bozzi et al. (BaBar Collaboration), 'First-Year Experience with the BaBar Silicon Vertex Tracker’, Nucl. Inst. \& Meth. A473 (2001), 7.

[29] C. Bozzi et al. (BaBar Collaboration), 'Status and Future Plans of the Babar Silicon Vertex Tracker', to be published in the proceedings of the $11^{\text {th }}$ International Workshop on Vertex Detectors, Kailua-Kona, Hawaii, November 3-8 2002.

[30] J. Beringer (for the BaBar Collaboration), 'CP Violation, B Mixing, and B Lifetime Results from the BaBar Experiment', Proceedings, $36^{\text {th }}$ Rencontres de Moriond on QCD and Hadronic Interactions, hep-ex/0105073, May 2001.

[31] B. Aubert et al. (BaBar Collaboration), Phys. Rev. Lett. 89 (2002) 201802.

[32] K. Abe et al. (BELLE Collaboration), Phys. Rev. Lett. 89 (2002) 071801.

[33] D. Williams (for the BaBar Collaboration), 'Charm Physics at Babar', 2002 Aspen Winter Conference on Particle Physics, Aspen, CO, February 3-9, 2002, http://hep.uchicago.edu/asp02/Talks/williams.pdf.

[34] D. Williams (for the BaBar Collaboration), 'D0 Mixing, Lifetime Differences, and Hadronic Decays of Charmed Mesons', Proceedings, $31^{\text {st }}$ International Conference on High Energy Physics, Amsterdam, The Netherlands, July 24-31, 2002. 
[35] W. Lockman (for the BaBar Collaboration), 'Charm Physics Results from BaBar', to appear in the Proceedings of the 2003 Lake Louise Winter Institute, Alberta, Canada, February 16-22, 2003.

[36] S. Godfrey and R. Kokoski, Phys. Rev. D43, 1679 (1991).

[37] M. Di Pierro and E. Eichten, Phys. Rev. D64, 114004 (2001).

[38] R. Ammar et al. (CLEO Collaboration), Phys. Rev. Lett. 71 (1993), 2391.

[39] M. S. Alam et al. (CLEO Collaboration), Phys. Rev. D52 (1995), 2661.

[40] B. Aubert et al. (BaBar Collaboration), Phys. Rev. Lett. 88 (2002), 101805.

[41] E. Golowich and S. Pakvasa, 'Phenomenological Issues in the Determination of $\Delta \Gamma_{D}{ }^{\prime}$, hep-ph/0102068, February, 2001.

[42] A. Ali and A. Ya. Parkhomenko, Eur. Phys. J. C23, 89 (2002).

[43] B. Aubert et al. (BaBar Collaboration), 'Search for the Exclusive Radiative Decays $B \rightarrow \rho \gamma$ and $B^{0} \rightarrow \omega \gamma$ ', Proceedings, $31^{\text {st }}$ International Conference on High Energy Physics, Amsterdam, The Netherlands, July 24-31, 2002.

[44] A. Ali and E. Lunghi, Eur. Phys. J. C26, 195 (2002). 


\subsection{ATLAS EXPERIMENT}

\subsubsection{ATLAS Overview}

Research on elementary particles has made outstanding progress, resulting in the development of the Standard Model, which explains not only the electromagnetic but also the weak and strong forces as arising due to gauge symmetry. The weak and electromagnetic forces, at first sight very different, are unified in the model. The discovery of the heavy analogs of the photon, the $\mathrm{W}$ and $\mathrm{Z}$, and, more recently, numerous detailed measurements of electron-positron collisions at the $\mathrm{Z}$ mass and higher energies have provided precise verification of the predictions of the Standard Model. However, the very nature of the electroweak unification, successful and beautiful as it is, indicates that there must be another equally important discovery still to be made, since the symmetry between the weak and electromagnetic forces would seem to imply that the $\mathrm{W}$ and $\mathrm{Z}$ gauge bosons should have exactly zero mass, like the photon. But the $\mathrm{W}$ and $\mathrm{Z}$ have masses close to 100 times the mass of the proton, so there must be a mechanism that breaks this symmetry. This electroweak-symmetry-breaking mechanism is also necessary to generate the quark and lepton masses. While there are several proposals for what this mechanism may be, we have only indirect evidence about it. However, we do know from general considerations that the physics involved must set in at a mass scale within an order of magnitude of the mass of the $\mathrm{Z}$, i.e., less than a TeV. To reveal the symmetry breaking mechanism, we therefore require the capability of producing particle systems of mass on the order of $1 \mathrm{TeV}$, with sufficient event rate to detect whatever new particles may exist, for example "Higgs bosons." This exploration at the energy frontier, however, goes well beyond searching for a particular particle to developing a deeper understanding of the origin of the many parameters that go into the Standard Model. Such understanding may involve the discovery of whole new families of particles. As an example, the discovery of supersymmetric particles is an attractive possibility; it typically requires much less luminosity than the Higgs discovery, if such particles are involved in electroweak symmetry breaking.

It is widely agreed that the exploration of the $1 \mathrm{TeV}$ mass scale is of the highest priority in particle physics, and that the high-luminosity Large Hadron Collider using proton-proton collisions, at the CERN laboratory in Geneva, Switzerland, is the most effective way to reach the necessary energy and luminosity. The energy available in the center-ofmass system will be $14 \mathrm{TeV}$. Two large general purpose detectors will study the events coming from the collisions. UCSC is heavily involved in one of the detectors, called ATLAS, where SCIPP participates in the design and construction of the large silicon strip inner tracking detector (SCT), and management of the strip and pixel system. This participation is based on almost 20 years of work on the relevant technology [1], including measurements on the behavior of the detectors and readout chips after irradiation [2], study of thermal failure modes [3], and simulations on the optimal geometrical detector configuration for the relevant physics measurements [4]. A. Grillo of SCIPP is responsible for management of all of the electronics (from front-end circuits to power supplies) for the complex SCT detector subsystem, an effort involving groups from eight different countries. Construction activities for the ATLAS detector are 
expected to last a couple more years, to be followed by installation at the collider. ATLAS should be ready for the first collisions in 2007.

Complementary to the SCT construction activities, the SCIPP group at CERN, led by A. Litke, in collaboration with Drevermann (CERN), has been developing a sophisticated, interactive global event visualization package called "Atlantis." This software package will be used for debugging/checking the reconstruction software (e.g. the Inner Detector track reconstruction software), to visualize backgrounds and detector performance, to investigate and visualize physics candidates, to provide figures for talks and publications, and to provide pictures and animations for public outreach. Atlantis will be particularly helpful during the commissioning phase of the SCT.

\subsubsection{ATLAS Construction Project}

SCIPP was one of the US institutions, which made the original proposal to DOE and NSF to join the ATLAS Collaboration in 1994, and to participate in the development and construction of the detector. Within the US-ATLAS organization, SCIPP is part of the Silicon Subsystem, which is made up of the Pixel Tracker, the Semi-Conductor Tracker (SCT) and the Readout Drivers (RODs) off detector readout electronics for both trackers. SCIPP's technical contributions to the construction project are focused on the SCT. That effort is led by A. Grillo and includes work on readout electronics, hybrids and modules, and system electronics. The ATLAS project is expecting the construction phase to last through 2005. Commissioning and pre-operations are planned to start in 2005, with first collisions scheduled for 2007.

\subsubsection{Level 2 Management of US ATLAS Silicon Subsystem}

Within the US ATLAS organization, A. Seiden is manager of the Silicon Subsystem. In this capacity he has oversight of the three components making up the subsystem (Pixels, SCT and RODs). There are eight US institutions working on this subsystem. The subsystem manager is responsible for managing the technical progress and budgets and representing the subsystem to the US Project Office at BNL. As the work transitions from construction to commissioning and finally research, the management structure is being maintained. New proposals for installation and for Maintenance and Operation have been developed between the Project Office and the subsystems for submission to DOE and NSF to carry the collaboration into the research phase.

\subsubsection{ATLAS SCT Readout Electronics}

The on-detector readout electronics for SCT were under development for many years by a collaboration of several institutes including SCIPP [6]. The difficulty of the requirements (e.g. low noise $<1800$ electrons, fast shaping time of 20 ns, low power consumption < 4mW/channel, radiation hardness to 10 Mrads) required developing an application specific integrated circuit (ASIC), which was designed by the collaboration. For this readout IC, three different architectures across four different technologies were sampled with each requiring its own custom circuit design [7]. Having selected an architecture and technology and completing a design, which met all the requirements, production orders were placed in July of 2001. 
The construction of the ASICs involves wafer fabrication by a selected vendor, electrical testing by the collaboration as well as quality assurance (QA) procedures to guarantee adherence to requirements. Orders were placed for 961 wafers representing 250,000 ICs. The testing of these circuits has required the shared work of three institutions, SCIPP, Rutherford Appleton Laboratory (RAL) in the UK and CERN. The testing has been done by similar test equipment at all three sites but ongoing care has been required to maintain identical test quality at all three [8]. Extensive cross-correlation of the three sites has kept this on track. Figure 2.3-1 shows one such correlation, the comparison of IC amplifier gains as measured on three production testers compared to the prototype tester. Given SCIPP's lead role in the electronics development, it was given responsibility for coordinating the manufacture and testing of all the ASICs. This included working with the vendor, managing the material flow through the testing sites as well as testing $65 \%$ of the wafers.

The actual testing at SCIPP has been performed by a team of five undergraduate students, led by our technician M. Wilder and post-doc F. Rosenbaum. One undergraduate completed his senior thesis on the correlation studies to qualify the three test sites. An administrative assistant manages the material flow and tracks the inventory for all three test sites.

This manufacturing period was scheduled to take 18 months ending at the end of calendar year 2002. Due to several issues with wafer manufacturing quality, the work has stretched out. As of April 2003, all of the wafers had been tested but several have been rejected for poor quality.. Fortunately, $87 \%$ of the needed chips have been identified as perfect through the testing process and there are more than enough ICs of slightly lower quality (i.e. 1 bad channel out of 128 in the IC) to complete the construction of the SCT. The use of the present ICs would reduce the overall individual hit efficiency of the tracker by approximately $0.1 \%$, much less than other losses already budgeted into the tracker design.

What remains to do in the ASIC manufacturing program is to conclude the negotiations with the vendor with regard to replacement of rejected wafers, test any further replacement wafers that they agree to deliver, select the necessary "1-bad-channel" chips to complete the needed inventory and distribute the remaining ICs to the hybrid assembly sites. This should be completed before the end of 2003. Also, during the hybrid and module assembly period, which will extend into mid-2004, there will be an ongoing need to correlate IC performance issues in modules with the test results previously obtained at wafer test. This analysis and expertise in IC performance issues will be provided as needed to the collaboration. 


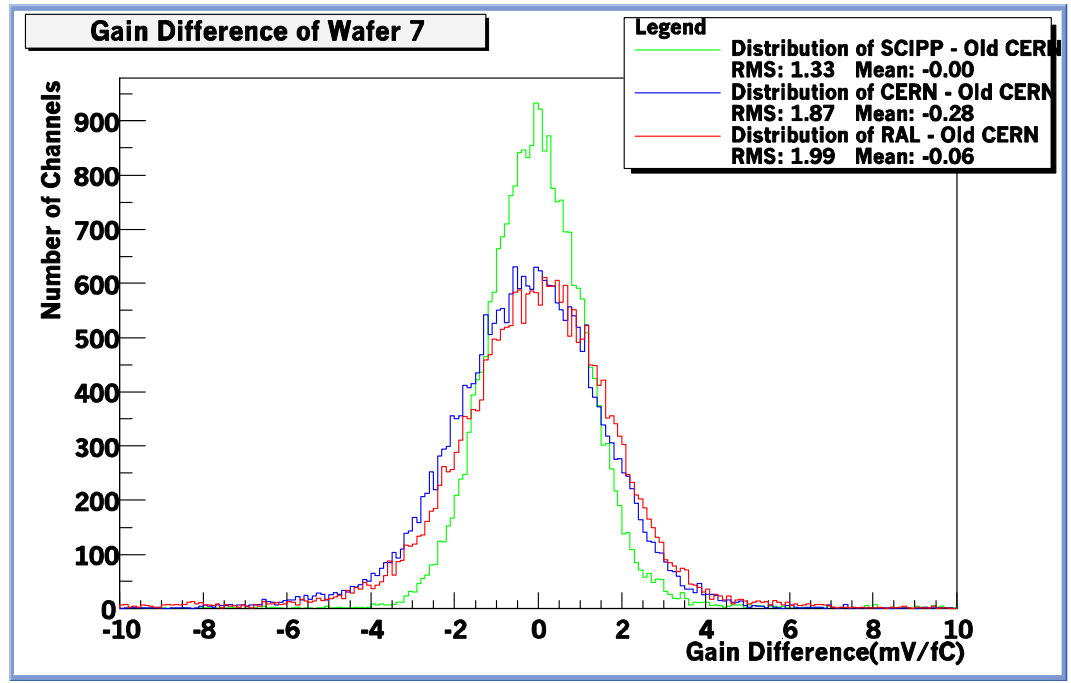

Figure 2.3-1: Gains Measured on Three Production Testers as Compared to the Prototype Tester

\subsubsection{ATLAS SCT Hybrid and Module Construction}

A major part of the SCT construction program is the assembly of detector modules. (See Figure 2.3-2.) This involves the assembly of 12 readout ASICs onto a high density hybrid circuit (gluing the 12 ICs onto the hybrid and wire bonding the ICs to the circuitry of the hybrid), gluing 4 micro-strip detector wafers together to form two back-to-back detector planes, mounting the hybrid across the detector planes and wire bonding the detector strips across pairs and to the input of the ASICs on the hybrid. In all there are over 5000 wire bonds per module. The positioning and gluing operations must hold tolerances of a few microns [9]. The total SCT will contain 2112 barrel modules and 1976 end-cap modules. The US has a commitment to build 30\% of the barrel modules. This is a shared responsibility of SCIPP and Lawrence Berkeley National Laboratory (LBNL).

\subsubsection{ATLAS SCT Systems Electronics}

In addition to the work focused on the specific on-detector readout IC, SCIPP has responsibilities with regard to the whole SCT electronics system. A. Grillo is the SCT Electronics Coordinator and E. Spencer is the SCT Electronics System Engineer. The

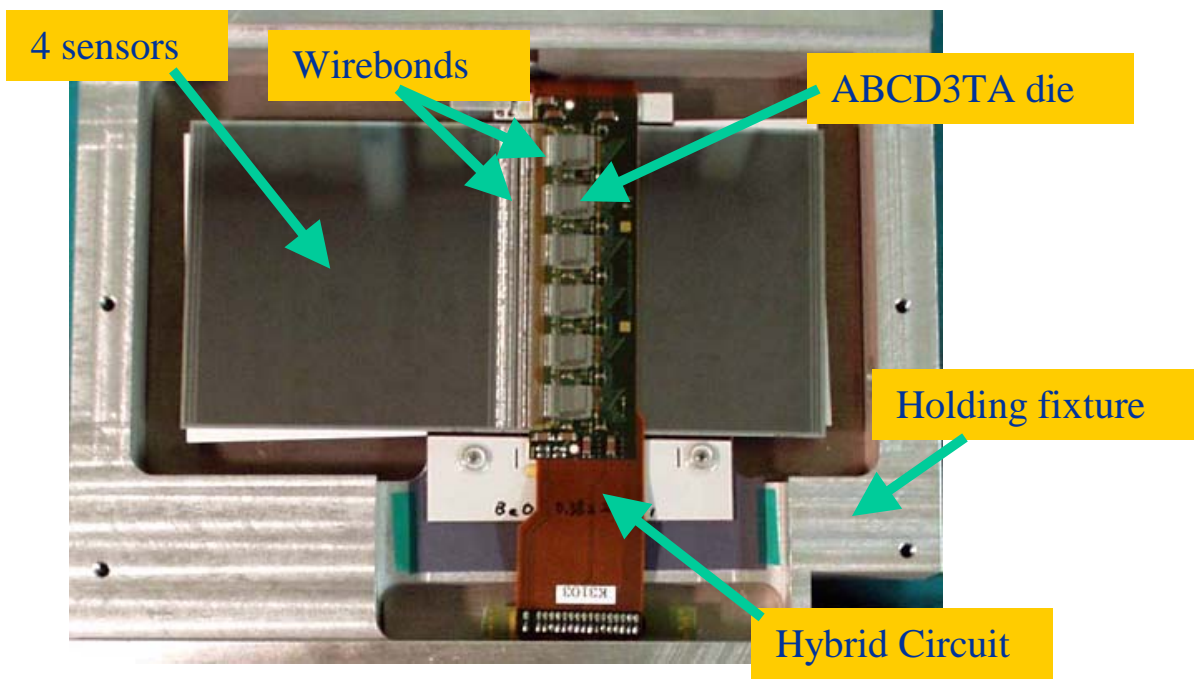

Figure 2.3-2: ATLAS-SCT Detector Module 


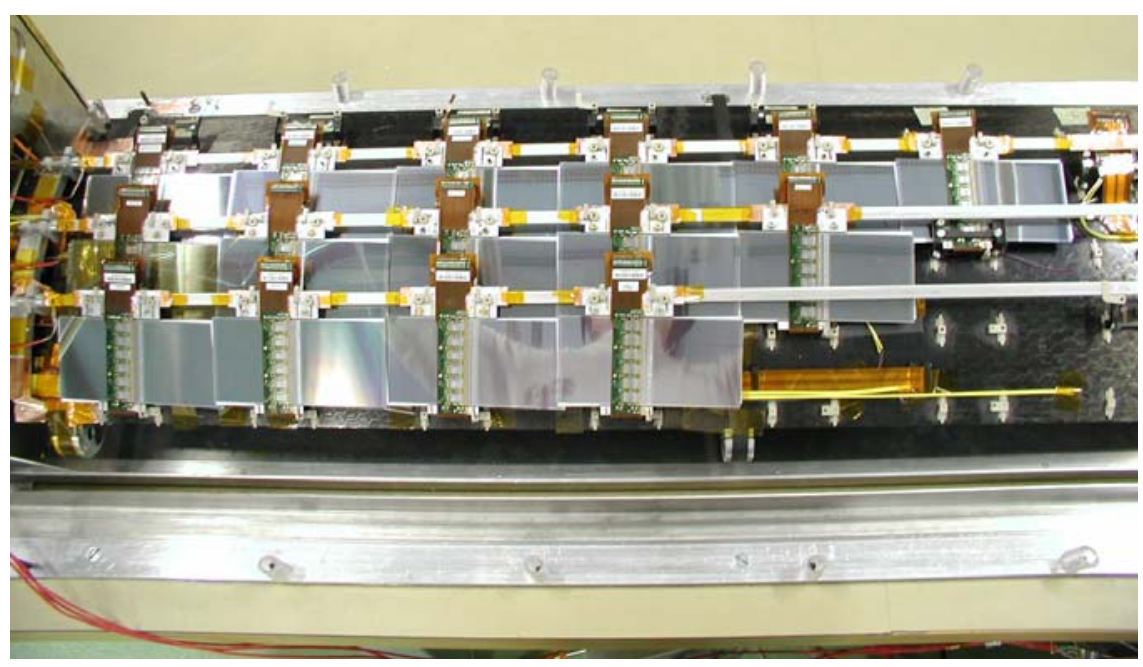

Figure 2.3-3: Barrel System Test at CERN with 15 Modules Mounted

coordination effort involves oversight of the developments of all the electronics components of the SCT, including the power supplies, the power distribution system, the optical data transmission, the off-detector readout (called RODs) and the detector control system (DCS). None of these components are SCIPP deliverables for the project. Rather, they are being developed by twelve other institutions in the US, Europe and Australia. The considerable electronics expertise at SCIPP is needed to tie the components together into a working system. Spencer, Grillo and D. Dorfan have experience with several successful experiments as well as in the electronics industry and form a good team for these electronics system issues.

A major concern is the grounding and shielding of the subsystem in order to minimize the interference from other subsystems and interference to other subsystems. The system tests at CERN, which were conducted prior to the Production Readiness Review for the readout IC, established proof of the basic operation of the system. Figure 2.3-3 shows the system test setup with fifteen modules arranged on the barrel sector. Since then tests have continued, which include attempts to add noise sources to the system and measure the susceptibility of the detector to pickup. In general, results have been very promising. Spencer is working with the mechanical engineering team at RAL to design the supports and thermal shielding in such a way as to maximize the electrical shielding and meet the performance requirements of the tracker.

This coordination work is being accomplished by regular teleconferences and occasional trips to Europe. As the project moves into the installation and commissioning stages, the need for this system engineering work will continue and intensify. More extended working periods at CERN are anticipated until the tracker is ready for data taking.

\subsubsection{ATLAS References}

[1] C. Adolphsen et al, Initial Beam Test Results from a Silicon-strip Detector with VLSI Readout, IEEE Trans. Nucl. Sci. NS-33, 57 (1986); A. Litke et al, A Silicon Strip Detector for the MARK II Experiment at the SLAC Linear Collider, Nucl. Instrum. 
Methods A265, 93 (1988); A. Litke and A. Schwarz, The silicon Microstrip Detector, Scientific American 272 (5), 56, (1995).

[2] D. Pitzl et al., Type Inversion in Silicon Detectors, Nucl. Instrum. Methods A311 98 (1992); H.J. Ziock et al, Temperature Dependence of Radiation Damage and its Annealing in Silicon Detectors, IEEE Trans. Nucl. Sci. 40344 (1993); H.F.-W. Sadrozinski, Depletion Voltage after Proton Irradiation with a Fluence of $10^{14} \mathrm{p} / \mathrm{cm}^{2}$, SCIPP Preprint 96/43 (1996); T. Dubbs, H.F.-W. Sadrozinski et al, Efficiency and Noise Measurements of Non-uniformly Irradiated Double-sided Silicon Strip Detectors, Nucl. Instrum. Methods A383 174 (1996).

[3] A. Seiden, Silicon Tracking at High Luminosity Colliders, IEEE Trans. Nucl. Sci. 44, 615 (1997); T. Dubbs et al., Thermal Measurements and Simulations with a Silicon Detector - Pyrolytic Graphite Sandwich, IEEE Trans. Nucl. Sci. 44, 743 (1997).

[4] A. Seiden, The SDC Silicon Tracker, IEEE Trans. Nucl. Sci. 41, 779 (1994); R. Sonnenblick et al., Electrostatic Simulations for the Design of Silicon Strip Detectors and Frontend Electronics, Nucl. Instrum. Methods A310, 189 (1991); E. Barberis et al., Capacitances in Silicon Microstrip Detectors, Nucl. Instrum. Methods A342, 90 (1994).

[5] For a summary and references see: "ATLAS Detector and Physics Performance Technical Design Report", volume 2, ATLAS Collaboration, CERN/LHCC/99-15 (25 May 1999).

[6] J. DeWitt et al., Signal-to-Noise in Silicon Microstrip Detectors with Binary Readout, IEEE Trans. Nucl. Sci. 42, 445 (1995); E. Spencer et al., A Fast Shaping Low-Power Amplifier-Comparator Integrated Circuit for Silicon Strip Detectors, IEEE Trans. Nucl. Sci. 42, 796 (1995); W. Dabrowski et al., A Single Chip Implementation of the Binary Readout Architecture for Silicon Strip Detectors in the ATLAS Silicon Tracker, London 1997, Proceedings of the Third Workshop on Electronics for LHC Experiments, London, September 1997 CERN/LHCC 97-60, pp. 153-157.

[7] W. Dabrowski et al., The ABCD Binary Readout Chip for Silicon Strip Detectors in the ATLAS Silicon Tracker, Rome 1998, Proceedings of the 4th Workshop on Electronics for LHC experiments CERN/LHCC 98-36, October 1998, pp. 175-179; A.A. Grillo, Overview of ATLAS Silicon Strip Readout System, Third International Meeting on Front End Electronics for High Resolution Tracking Detectors, Taos, November 1997; W. Dabrowski et al., Radiation Hardiness of the ABCD Chip for the Binary Readout of Silicon Strip Detectors in the ATLAS Semiconductor Tracker, Snowmass, Colorado, USA 1999, Proceedings of the $5^{\text {th }}$ Workshop on Electronics for LHC Experiments, CERN 99-09, October 1999, pp.113-117; W. Dabrowski et al., Progress in Development of the Readout Chip for the ATLAS Semiconductor Tracker, Krakow, Poland 2000, Proceedings of the $6^{\text {th }}$ Workshop on Electronics for LHC Experiments, CERN 2000-010 October 2000, pp. 115-119.

[8] F. Anghinolfi et al., ASIC Wafer Test System for the Atlas Semiconductor Tracker Front-End Chip, IEEE Trans. Nucl. Sci. 49, 1080, (2002).

[9] T. Kondo et al., Construction and Performance of the Atlas Silicon Microstrip Barrel Modules, Nucl. Instrum. Meth. A485, 27, (2002). 


\subsection{Gamma Large Area Space Telescope}

\subsubsection{GLAST Overview}

The Gamma-ray Large Area Telescope (GLAST) is a satellite mission that will orbit the first survey-class instrument to view the universe using gamma rays in the $>20 \mathrm{MeV}$ energy range. It is the follow-up to the highly successful EGRET experiment on the Compton Gamma-Ray Observatory (CGRO) [1][2], which opened up the high-energy gamma-ray end of the electromagnetic spectrum to detailed investigations. In February 2000, NASA selected the proposal of our collaboration to build and operate an instrument with sensitivity more then 50 times that of EGRET and a potential lifetime several times greater. The collaboration is a partnership between NASA, DOE, and foreign collaborators, with a total of 30 institutions worldwide. In the following we detail the GLAST science, the instrument we are building, and the role of the SCIPP scientists in the development of the GLAST program.

\subsubsection{GLAST History}

The GLAST Project was conceived in 1992 after the first data from the EGRET experiment onboard the Compton Gamma Ray Observatory brought much excitement to the astrophysical community. There was an amazing gamma-ray sky being revealed, filled with transient phenomena and a multitude of sources both close by within our galaxy as well as at cosmological distances.

The original design of the instrument was due to $\mathrm{W}$. Atwood, who realized that environmental challenges of a space-based experiment narrowed the choice of tracking technologies to just a few. This was contemporaneous with the conceptual design efforts for the SSC and LHC experiments, where large arrays of silicon strip detectors were being seen as possible and within reach with regard to cost and complexity. Hence this technology was selected to do the tracking in GLAST. The Calorimeter similarly reflects contemporary thinking: CsI crystals read out by photodiodes. The design has evolved since then, but only in relatively small ways. Principal among these was the addition of a plastic scintillation veto system (thus avoiding the expense of more silicon layers) to void triggers caused by cosmic rays. Atwood performed essentially all of the formative Monte Carlo studies, which were used to refine the design from its inception up until 1998.

However the main challenge of adapting the silicon-strip tracking technology to space, was dealing with the large channel count inherent in this approach. Electric power on a spacecraft is at a premium, and even if it were readily available, shedding the resulting waste heat presents its own problems. At the time of conception the lowest power frontend electronics for silicon strip detectors operated at a few milliwatts per channel. With an anticipated channel count of about 1 million, a much leaner solution was necessary. R. Johnson took up this challenge while still helping with the design and development of the front-end electronics for the silicon strip vertex tracker presently in use in the BaBar experiment at SLAC. By optimizing the design to the GLAST requirements, taking advantage of the fact that the GLAST application could have a slower response and lower 
clock speed, Johnson was able to beat the single channel power down by more than an order of magnitude. In addition, he achieved such a low noise level in his design that it became possible to increase the length of the silicon strips, thereby reducing the channel count.

H. Sadrozinski early on began organizing and directing inquiries to industry about purchasing the nearly hundred square meters of silicon strip detectors that GLAST would require. His contacts in Japan and experience in working on large silicon-strip systems resulted in successful negotiation of a contract presently being executed by Hamamatsu Photonics Inc.

The SCIPP group under the leadership of Johnson produced all of the GLAST Tracker prototype devices. The first beam test device, consisting of 12 planes of single SSDs and custom relectronics [3], was successfully tested at SLAC in both electron and photon beams in 1997 and conclusively demonstrated the performance as predicted by the Monte Carlo simulations [1]. Following this, an almost complete prototype Tracker tower module was built at UCSC. It is known as the Beam Test Engineering Model (BTEM) and was tested at SLAC in 1999 and refurbished and flown on a balloon in 2001 [6][7][8]. Both of these tests again validated the GLAST tracker concept and demonstrated the robustness of the technology and construction.

\subsubsection{GLAST Science}

Our understanding of the universe has experienced a revolution in the last few years. A major contributor has been the observations by EGRET of high-energy gamma rays from blazars, pulsars, and unidentified sources, of delayed emission from gamma-ray bursts and solar flares, and of the diffuse gamma radiation. Gamma rays provide a unique window into a variety of violent, transient processes, and they have the distinct advantage over other high-energy cosmic rays of pointing back to their source, thus allowing identification of counterparts in other wavelength bands, such as the radio, optical and $x$ ray. The GLAST end of the electromagnetic spectrum is where the non-thermal processes show themselves and hint at more exotic mechanisms at work transforming gravitational and/or rotational energy into high-energy particle acceleration.

EGRET's discoveries have posed as many new questions as they have given answers. Many blazars are detected almost exclusively in a flaring state, but what is their quiescent emission? Afterglow has been found in both $\gamma$-ray bursts and solar flares, lasting many times longer than anyone had expected. The GeV afterglow from GRB940217 hints at a powerful accelerator mechanism at work long after the initial explosion, but how common is this situation and what is its explanation? New source classes of $\gamma$-ray blazars and radio-quiet $\gamma$-ray pulsars have been discovered, with hints of others to come (millisecond pulsars, radio galaxies, supernova remnants, $x$-ray binaries). In fact, of the 271 gamma-ray point sources discovered by EGRET, 170 remain unidentified. GLAST will discover of the order 10,000 new sources, with much better localization than possible with EGRET, which will allow new systematic and statistically powerful approaches to all of these questions.

Some important questions facing us that can be addressed by GLAST are 
- What are the mechanisms of particle acceleration in the Universe?

- What are the origins of Gamma-Ray Bursts and other transients?

- What are the unidentified EGRET sources, and what new classes of gamma-ray sources can be observed?

- What are the distributions of mass and cosmic rays in our galaxy and nearby galaxies?

- How can high-energy gamma rays be used to probe the early universe?

- What is the nature of dark matter?

To make significant progress in understanding the high-energy sky, a highly capable instrument is required with

- maximal sensitivity, to image a large number of sources and look deeper into space,

- good angular resolution for source localization, to enable or facilitate multiwavelength studies and to minimize background and source confusion,

- a broad field-of-view to monitor variability and detect transients, providing approximately continuous monitoring of the entire sky,

- good calorimetry over an extended energy band to study spectral breaks and cut-offs,

- good calibration, long-term stability and lifetime.

GLAST will provide overlap in the $50 \mathrm{GeV}$ to $1 \mathrm{TeV}$ range with ground-based telescopes, with approximately $10 \%$ spectral resolution, thereby providing these experiments with an absolute energy calibration and allowing us to explore together a greatly expanded dynamic range with well-matched capabilities.

The main GLAST science objectives may be categorized into four themes:

\subsubsection{Understand the mechanisms of particle acceleration in Active Galactic Nuclei (AGNs), Pulsars, and Super Nova Remnants (SNRs).}

This understanding is a key to solving the mysteries of the formation of jets, the extraction of rotational energy from spinning neutron stars, and the dynamics of shocks in SNRs. GLAST will detect as many as $10^{4}$ extragalactic sources as well as hundreds of galactic sources during its first two years of operation. The large field of view (FOV) and good energy measurement capability will allow detailed comparisons with models of AGN jets over a range of flare intensities two orders of magnitude larger than EGRET could detect. Figure 2.4-1 shows the emission spectrum of Mrk 501, an especially powerful blazar, with simulated GLAST data superposed. The large FOV and sensitivity of GLAST will allow detailed study of a wide variety of pulsars $(>50)$ during scanning observations. Good calorimetry for measuring spectral roll-offs above $1 \mathrm{GeV}$, and phaseresolved spectra, uniquely probe cascade models. The expected shock acceleration of cosmic-ray nuclei in SNRs will be observed for the first time by resolving the acceleration site both spatially and spectrally in more than 10 nearby SNRs. 


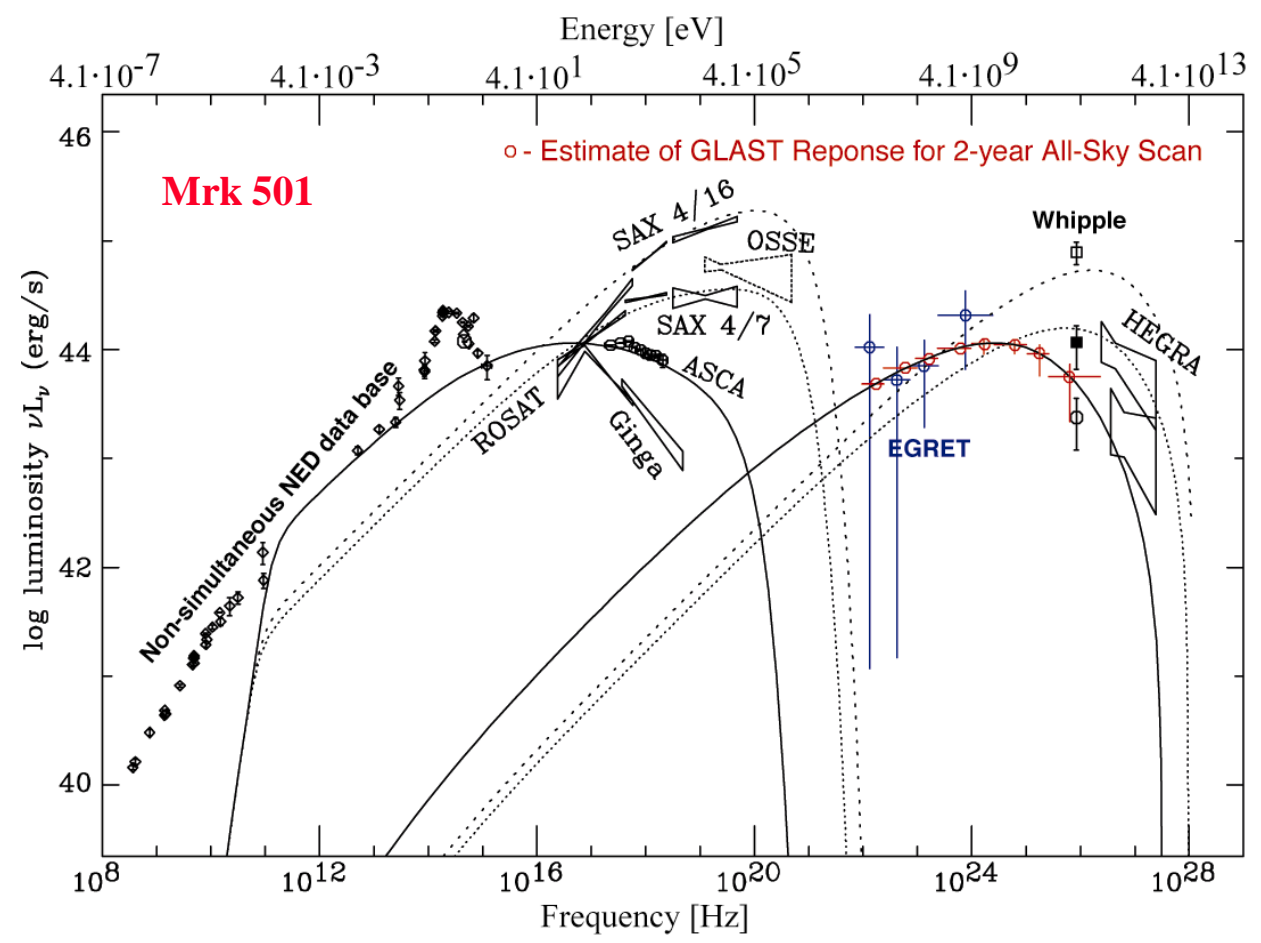

Figure 2.4-1. Multi-wave Observation of the Emission of AGN Markarian 501.

\subsubsection{Resolve the gamma-ray sky: unidentified sources and diffuse emission.}

Interstellar emission from the Milky Way and a large number of unidentified sources are prominent features of the gamma-ray sky. GLAST will help determine the identity of the latter by source localization to one arc minute and through searches for time-variability or pulsations, including high-sensitivity blind searches for periodicity typical of pulsars and binary systems. Recall that the Geminga pulsar shows variability only in $x$-rays and gamma rays. There may be more Geminga-like pulsars to be found. With its improved angular resolution, GLAST will also address cosmic-ray propagation in the interstellar matter and magnetic field of the Milky Way on kpc and sub-kpc distance scales. On larger scales GLAST will open up studies of cosmic-ray production and propagation in nearby galaxies and clusters of galaxies.

\subsubsection{Determine the high-energy behavior of gamma-ray bursts and transients.}

Variability has long been a powerful method to decipher the workings of objects in the universe on all scales. Variability is now recognized as a central feature of the gammaray sky. In scanning mode, GLAST will detect the weakest EGRET sources $\left(>6 \times 10^{-8}\right.$ photons $\mathrm{cm}^{-2} \mathrm{~s}^{-1}$ ) in a single day with $>5 \sigma$ significance. Brighter AGN flares will be detected within minutes. On shorter-duration timescales, GLAST will detect about 200 gamma-ray bursts per year, providing localization to better than 3 arc minutes for $25 \%$ of these bursts, and will provide spectra up to $100 \mathrm{GeV}$, with less than $20 \mu \mathrm{s}$ of deadtime after triggering (compared with $100 \mathrm{~ms}$ for EGRET, see Figure 2.4-2). The wide field of view will also allow study of delayed high-energy emission and how it relates to the afterglow at lower energies. We anticipate that the correlation between GLAST sightings 


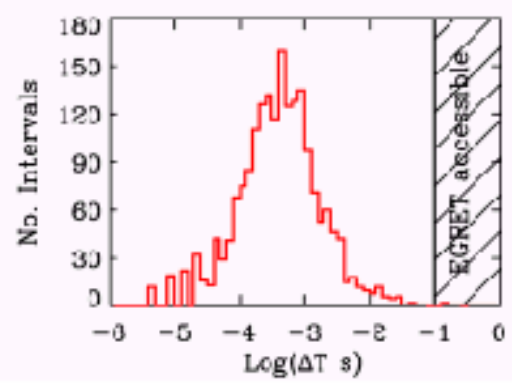

Figure 2.4-2. Simulated time interval between gamma rays from about 200 GRB expected for GLAST, enabling observation of substructure on the sub-millisecond scale in the $>20 \mathrm{MeV}$ energy band.

and other instruments such as LIGO could yield new understanding on the mechanisms that produce these occurrences.

\subsubsection{Probe dark matter and the early Universe.}

Observations of gamma-ray AGN serve to probe super-massive black holes through jet formation and evolution studies, and provide constraints on the star-formation rate at early epochs through absorption over extragalactic distances. The "absorption" mechanism is posited to be the result of very high-energy gammas creating $\mathrm{e}^{+} \mathrm{e}^{-}$pairs off the background light pool present in the intervening space. For AGNs with red shifts around $\mathrm{z}=2$, models indicate that the resulting high-energy cut-offs occur below $50 \mathrm{GeV}$ and are well suited to the GLAST energy range. In addition, this co-aligned beam of charged particle pairs may provide a probe of the inter-galactic magnetic fields through deflection as well as timing delays. The presence of such effects is revealed by these charged particles subsequently up-scattering low or extremely-low energy photons (e.g.

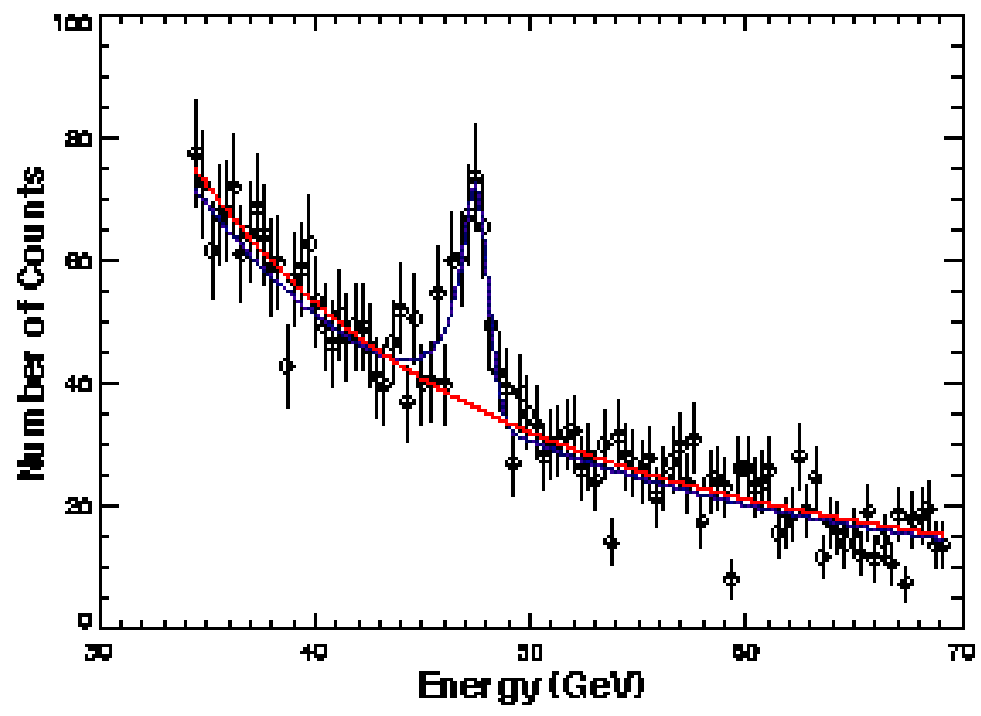

Figure 2.4-3. Simulation of the annihilation of supersymmetric particles of about $50 \mathrm{GeV}$, resulting in quasi-monochromatic lines. An energy resolution of $3 \%$ is assumed. 
the CMB) to energies within the GLAST range.

There are also possibilities for observing monoenergetic gamma-ray "lines" above $30 \mathrm{GeV}$ from super symmetric dark matter interactions (Figure 2.4-3); detecting decays of relics from the very early Universe, such as cosmic strings or evaporating primordial black holes; or even using gamma-ray bursts to detect quantum gravity effects.

\subsubsection{GLAST Instrument}

GLAST is a high-energy pair-conversion telescope. Our design has drawn upon the extensive experience of EGRET, as well as other space-based experiments and numerous high-energy accelerator-based experiments. Detailed simulations, trade studies and technology development have validated a modular instrument design composed of three detector subsystems and a modern data-acquisition system (Figure 2.4-4).

\subsubsection{Precision converter-tracker (TKR)}

Incident photons convert in one of the 16 layers of tungsten converter, and the resulting $e$-and $e+$ particles are tracked by single-sided silicon-strip detectors (SSD's) through successive planes (Figure 2.4-5) [5]. The pair conversion signature is also used to help reject the much larger background of charged cosmic rays. The high intrinsic efficiency and reliability of the silicon-strip technology enables straightforward event reconstruction and an excellent point spread function (PSF) with small tails. These ease-of-use properties along with the long life expectancy of this technology will maximize the mission's science return for observers.

\subsubsection{Calorimeter (CAL)}

CsI(Tl) bars, arranged in a segmented manner, give both longitudinal and transverse information about the energy deposition pattern. The depth of the calorimeter is 8.5 radiation lengths (r.l.) giving a total instrument depth of 10.1 r.l. for head-on trajectories. The depth and segmentation enable the high-energy reach of GLAST and contribute significantly to the background rejection.

\subsubsection{Anticoincidence Detector (ACD)}

The ACD array of plastic scintillator tiles provides most of the rejection of charged particle backgrounds. Its segmentation minimizes the "backsplash" self-veto that affected EGRET above a few $\mathrm{GeV}$.

\subsubsection{Data Acquisition System (DAQ).}

This system collects the data from the subsystems, implements the multi-level event trigger, and provides an on-board science analysis platform to detect transients, thus allowing for notification to other experiments both in space as well as on the ground. 
Incident particles successively encounter the ACD, the TKR and the CAL. The overall aspect ratio of the instrument (height/width) is $\sim 0.4$, allowing a large field of view and ensuring that nearly all showers initiated in the TKR will pass into the CAL for energy measurement. The instrument design is modular, with the TKR, CAL and associated DAQ modules forming an array of 16 identical towers supported by a low-mass grid structure. Modularity provides ample redundancy and offers many benefits, including:

- Simplified event reconstruction.

- Ease of fabrication, assembly and integration.

- Significant risk reduction through early testing at flight scale.

- Comprehensive pre-flight calibration studies can be done in detail over many months using a subset of towers during production without impact on the overall production schedule.

\subsubsection{GLAST Collaboration}

GLAST is an international space mission to be launched in 2007. It is a collaboration between DOE, NASA and several international partner agencies. An important aspect is the introduction of novel technologies developed in HEP, for example the use of megachannel solid-state detector and electronics systems. As in HEP, this increases the quality of the data and reduces the risk of critical failures.

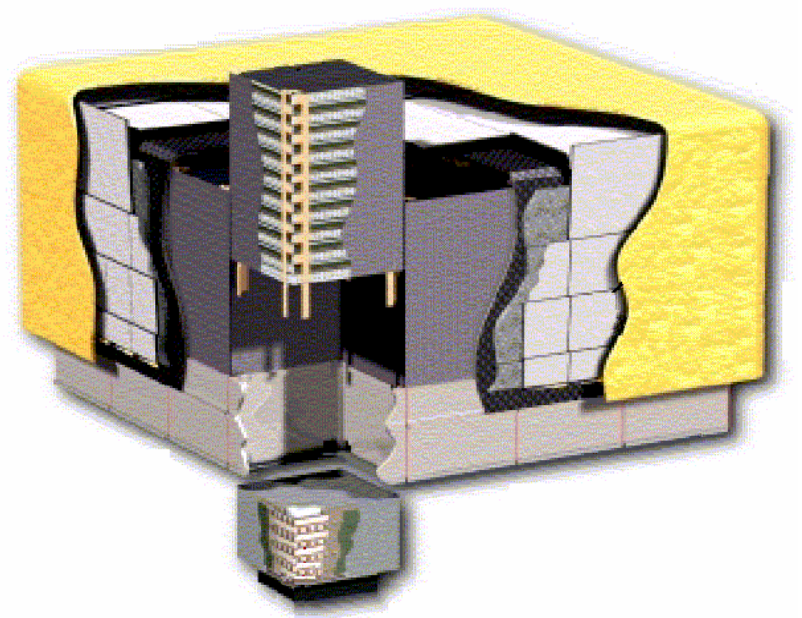

Figure 2.4-4. The GLAST Instrument. One of the 16 towers is shown disassembled into separate tracker and calorimeter modules. The segmented anti-coincidence detector and the thermal blanket (outermost layer) are shown cut away. 


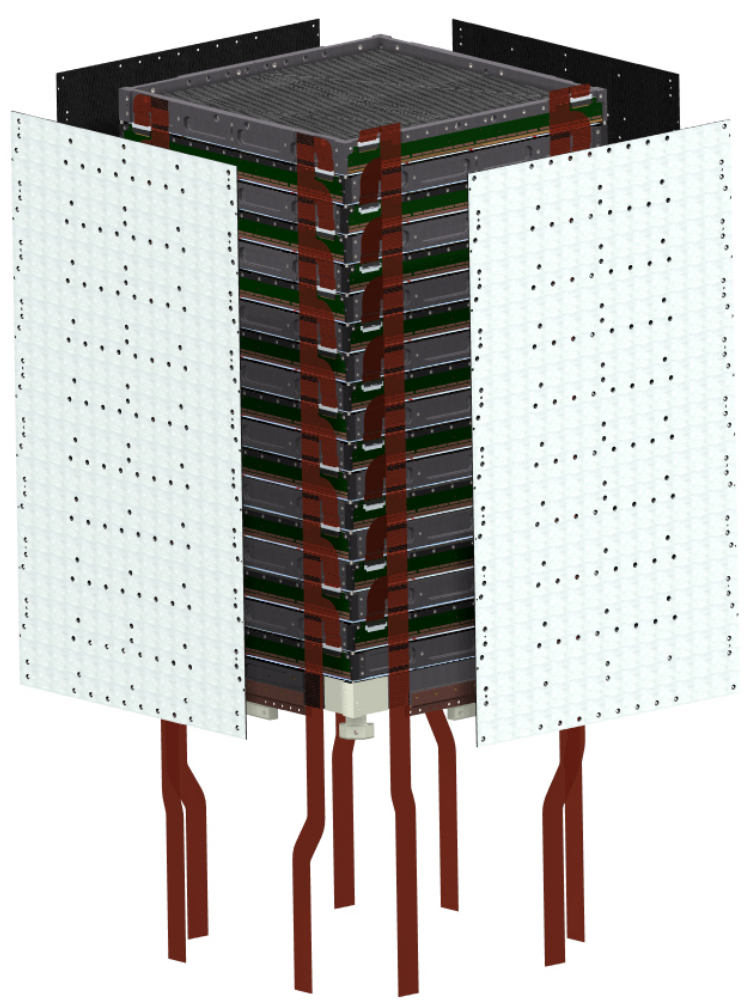

Figure 2.4-5. CAD view of a single Tracker module, with the sidewalls removed to show the electronics boards and readout cables.

GLAST is organized into the subsystems representing the principal functional components of the instrument. Because they are physically separate, with clean interfaces, they can and are developed quite autonomously. In addition, funding sources map naturally onto these subsystems. SCIPP is in charge of the Tracker subsystem, and with DOE funding has overall responsibility for delivering the silicon tracker. Our Japanese collaborators provide more than half of the Tracker silicon detectors, and our Italian collaborators at INFN contribute the remainder of the silicon detectors, fabricate the mechanical structures, and assemble the trays and tower modules (16 flight and 2 qualification units/spares). The Naval Research Lab (NRL), France and Sweden are providing the CsI calorimeter. The ACD is being built by Goddard Space Flight Center (GSFC), and the DAQ is provided by SLAC and NRL. The DOE commitment is based on a detailed proposal submitted in 1998 that was approved after being extensively peerreviewed, for example by SAGENAP.

R. Johnson is the tracker subsystem manager, and H. Sadrozinski is the tracker scientist. W. Atwood, R. Johnson and H. Sadrozinski are members of the 17 member GLAST LAT Senior Science Advisory Committee (SSAC). W. Atwood, R. Johnson, H. Sadrozinski and T. Schalk are members of the group of 64 GLAST Co-Investigators (Co-I), and J. Primack and S. Woosley are Associate Scientists. S. Thorsett is a member of the GLAST Science Working Group. The latter two are members of the Astrophysics Dept. at UCSC. 


\subsubsection{GLAST References}

[1] D.J. Thompson, et al., ApJS 86, p. 629, 1993.

[2] R.C. Hartman, et al., ApJS 123, p. 79, 1999.

[3] R.P. Johnson et al., "An Amplifier-Discriminator Chip for the GLAST Silicon-Strip Tracker,” IEEE Trans. Nucl. Sci. 45 (1998) 927.

[4] W.B. Atwood, et al., "Beam Test of Gamma-Ray Large Area Space Telescope Components,” Nucl. Instr. Meth. A446 (2000) 444.

[5] J.A. Hernando et al., "The Silicon Tracker/Converter for the Gamma-Ray Large-Area Space Telescope,” Nucl. Instr. Meth. A435 (1999) 224.

[6] E. Atwood, et al., Nucl. Instr. Meth. A457, p. 126, 2001.

[7] P. Allport, et al., Nucl. Instr. Meth. A466, p. 376, 2001.

[8] E. do Couto e Silva, et al., Nucl. Instr. Meth. A474, p. 19, 2001 


\subsection{The SCIPP Linear COLlider PROgRAM}

\subsubsection{Introduction}

In the autumn of 2001, the High Energy Physics Advisory Panel (HEPAP) to the Department of Energy gave the following recommendation regarding the question of U.S. participation in the development of a high-energy electron-positron Linear Collider:

"We recommend that the highest priority of the U.S. program be a high-energy, highluminosity, electron-positron linear collider, wherever it is built in the world. This facility is the next major step in the field, and should be designed, built and operated as a fully international effort."

This recognition of the central role a future Linear Collider will play in the field of particle physics is in support of a larger, world-wide consensus that identifies the Linear Collider as the clear choice for the next major worldwide collider facility.

SCIPP physicists have been increasingly active on Linear Collider development over the last 10 years or so. Areas of interest include the physics and machine requirements for an $\mathrm{e}^{-} \mathrm{e}^{-}$Linear Collider option, research and development towards the optimization of the central tracker for a Linear Collider detector, and physics studies of QCD, Supersymmetry, and alternative symmetry-breaking signals that might be observed at a high energy $\mathrm{e}^{+} \mathrm{e}^{-}$colliding beam facility. Currently, an active group of approximately ten SCIPP faculty, post-doctoral fellows, graduate and undergraduate students, and technical staff lend their expertise or work part-time on Linear Collider R\&D issues.

\subsubsection{The Linear Collider Physics Program}

The corpus of precision electroweak data, underscored by theoretical considerations, strongly suggest that a light Higgs awaits discovery in the near future. In its most basic terms, the goal of the Linear Collider (LC) physics program will be to deepen and enrich the exploration of the electroweak symmetry-breaking mechanism that will have begun at the Large Hadron Collider (LHC) towards the end of this decade.

With its well-defined initial state and precision detectors, the LC will be an optimal facility for the precise measurement of Higgs Boson properties. Exacting branching fraction measurements, essential to the discrimination between the minimal Standard Model and other Higgs sector scenarios, will be made possible by state-of-the-art charged-track vertexing and momentum reconstruction, combined with precise energyflow calorimetry. Figure 2.5-1 shows the expected precision on Higgs branching fractions as a function of Higgs mass, assuming an integrated luminosity of $500 \mathrm{fb}^{-1}$ at a cms energy of $350 \mathrm{GeV}$, corresponding to 1-2 years of running at design luminosity [1]. 


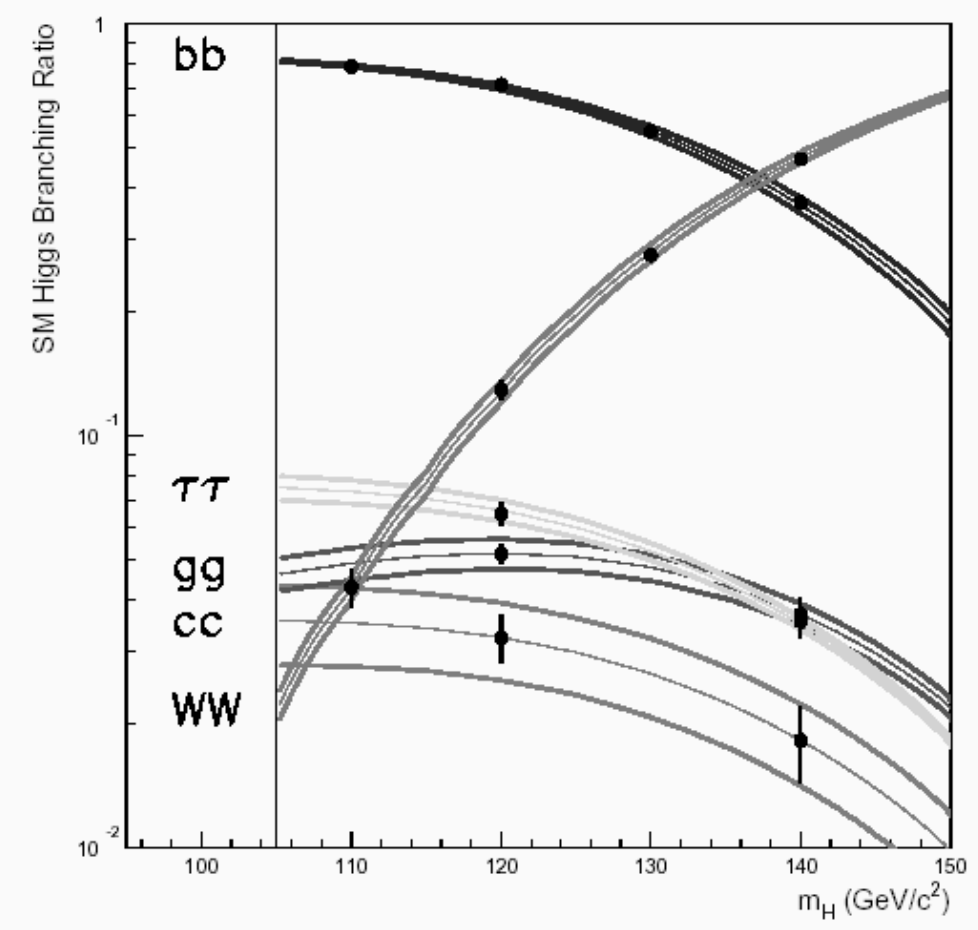

Figure 2.5-1: Expected branching fraction accuracy versus Higgs mass for a $500 \mathrm{fb}^{-1}$ sample. Bars show experimental uncertainty; bands show current Standard Model theoretical uncertainty.

The primary mode of Higgs production at intermediate energy is via radiation from an schannel $\mathrm{Z}^{0}$ boson, as shown in Figure 2.5-2. With precise tracking, this mode can be reconstructed in a model-independent way from the mass spectrum of the system recoiling from the leptonic decay channels of the $Z^{0}$, enabling a precise measurement of the $\mathrm{Z}^{0}$-Higgs coupling independent of the decay properties of the Higgs. If this Higgs is to be responsible for the generation of the $\mathrm{Z}^{0}$ mass, then it must be found that

$$
g_{Z h}^{2}=\frac{4 M_{Z}^{2}}{v^{2}}
$$

where $g_{Z h}$ is the value of the $\mathrm{Z}^{0}$-Higgs coupling and $v=246 \mathrm{GeV}$ is the well-known vacuum expectation value of the Higgs field. A run of $500 \mathrm{fb}^{-1}$ will test this relation to 2$3 \%$, providing a test of the tenets of electroweak symmetry breaking that goes right to the heart of the theory. This is another of several examples that underscore the essential contributions that a Linear Collider can make towards the understanding of the mechanism of electroweak symmetry breaking. 


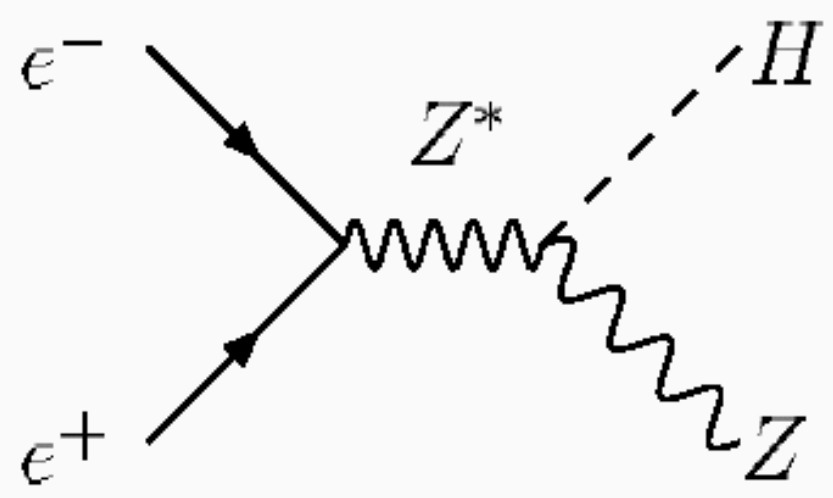

Figure 2.5-2: The 'Higgstrahlung' process that is the primary mode of Higgs production Higgs near threshold.

Should the Higgs mechanism not be nature's answer to the origin of the masses of the fundamental particles, unitarity requires that some other mechanism must modify the effective $W$-W-boson coupling at high energies. Although the direct observation of the source of these effects (e.g. high-mass resonances) may be out of reach of the LHC and LC, a precise study of $e^{+} e^{-} \rightarrow W^{+} W^{-}$production at the LC would be sensitive to the anomalous spacetime structure of the triple-boson coupling that would expose such effects, and provide essential guidance for future accelerator efforts - even for the initial $500 \mathrm{GeV}$ center-of-mass energy of the machine.
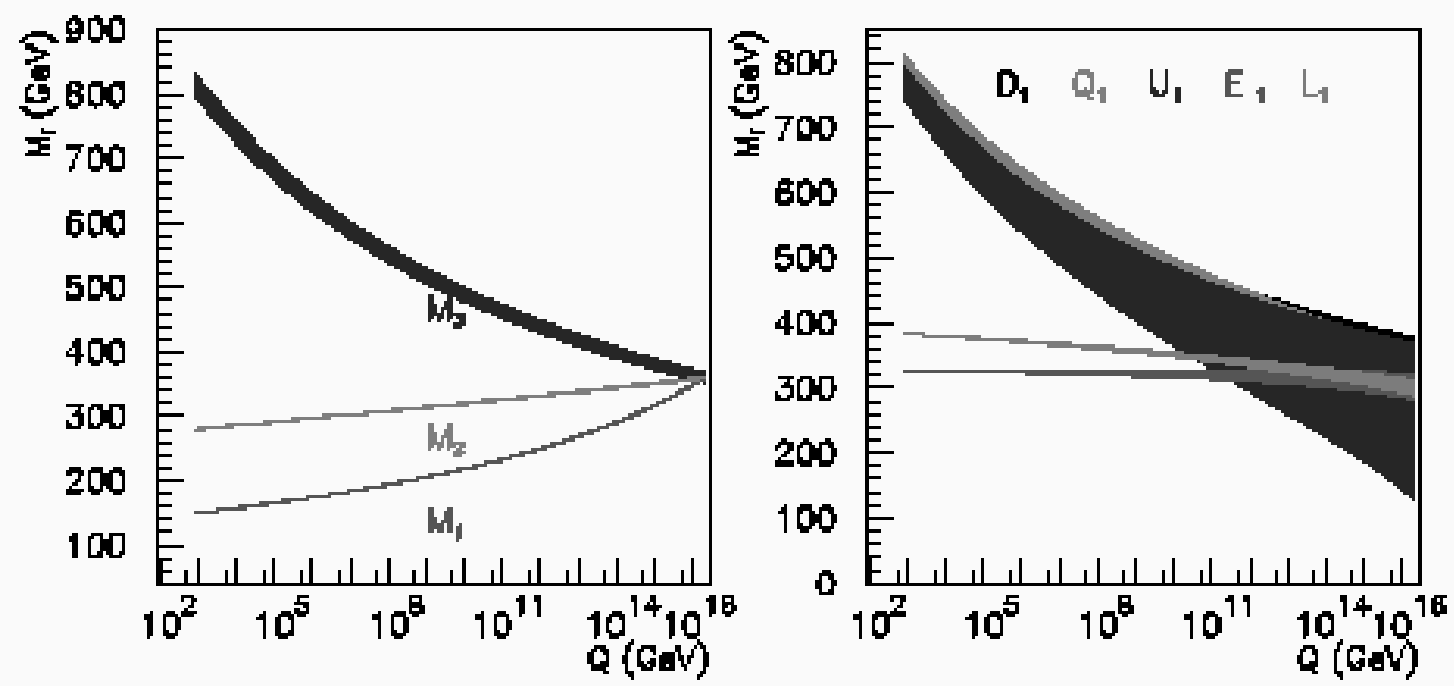

Figure 2.5-3: Typical MSUGRA mass constraints available after operation of the Linear Collider at $E_{c m}=1 \mathrm{TeV}$. 
Thinking beyond the electroweak scale, to that of Grand Unification, the Linear Collider is an ideal facility for the study of accessible supersymmetric states. In addition to states from the enhanced SUSY Higgs sector, most supersymmetric scenarios place several superpartners - sleptons and light gauginos -- within reach of the Linear Collider program. Definitive knowledge of the $e^{+} e^{-}$initial state, including electron polarization, will allow for the precise kinematic reconstruction of superparticle masses, and the potential to probe the nature of supersymmetry breaking and other GUT-scale physics. Figure 2.5-3 [2] shows the renormalization-group evolution of gaugino and sfermion masses, for a supergravity-inspired (mSUGRA) SUSY-breaking scenario. While not all sparticle masses would be accessible to a $1 \mathrm{TeV}$ Linear Collider, those that are would be measured with great precision. As shown in Figure 2.5-3, this precision would be essential to identifying the relationships that reveal the underlying GUT-scale physics -in this example, the universality of gaugino and sfermion masses that characterize mSUGRA models. Again, the ability to perform precision measurements is a hallmark of the Linear Collider physics program, and one that holds great promise for the advancement of our understanding of nature.

\subsubsection{SCIPP Contributions to Linear Collider Development}

\subsubsection{The SCIPP Linear Collider Group}

A number of SCIPP faculty, staff and students are active at various levels on the Linear Collider. Faculty members include David Dorfan, Clem Heusch, Hartmut Sadrozinski, Bruce Schumm, and Abe Seiden. Staff members active on the LC include Senior Research Physicist Alex Grillo and electrical engineer Ned Spencer. A new post-doc, Gavin Nesom (currently finishing his degree at Oxford) will join the group in May, replacing post-doctoral fellow Wolfgang Walkowiak, who recently left the group to take a permanent position at the University of Siegen in his native Germany (this position is designated as 1/2-time each on the LC and BaBar). Second-year graduate student Christian Flacco is currently devoting $100 \%$ of her research time to LC R\&D; she will begin as a thesis student on BaBar towards the beginning of the next academic year. One undergraduate student (Sharon Gerbode) is completing her undergraduate senior thesis this academic year on LC physics simulation; three more who will finish their senior theses next academic year (Heath Holguin, Paul Mooser, and Adam Pearlstein) have just joined the simulation effort.

\subsubsection{Exploration of the $e^{-} e^{-}$Option}

The recent worldwide consensus on the identification of the Linear Collider as the next major accelerator project has been formally accompanied by the statement: 'The baseline machine should have the option of $e^{-} e^{-}$collisions.' This is a great success for SCIPP efforts over the years, which have concentrated on investigating both the physics promise of the $e^{-} e^{-}$mode and its technical compatibility with the $e^{+} e^{-}$accelerator and 
IR/detector. The biannual International Electron-Electron Linear Collider Workshops organized by SCIPP on the UCSC campus have been instrumental in bringing both the scientific urgency and the technical feasibility aspects to the point that helped convince our community that this is a baseline project for the Collider; and the proceedings volumes of this series is generally seen as defining the field. 


\subsubsection{Linear Collider References}

[1] M. Battaglia, hep-ph/9910271, October, 1999; published in the proceedings of the $4^{\text {th }}$ International Workshop on Linear Collider, Sitges, Spain, April 28 - May 5, 1999.

[2] G. A. Blair, W. Porod, and P. M. Zerwas, Phys. Rev. D63 017703 (2001).

[3] A wealth of information about the machines, physics, and detectors of the Linear Collider program can be found in the major documents recently provided by the European and North American, and Asian working groups. The TESLA TDR can be found at http://www.desy.de/ lcnotes/tdr/, the North American Resource Book for Snowmass 2001 can be found at http://www.slac.stanford.edu/pubs/slacreports/slac-r570.html, and the Asian treatise on experimentation at the JLC can be found at http://acfahep.kek.jp/acfareport/index.html.

[4] See http:://scipp.ucsc.edu/ schumm/lctrack

[5] See http://blueox.uoregon.edu/ jimbrau/LC/ALCPG/

[6] T. Abe et al., (American Linear Collider Working Group), 'Linear Collider Physics -Resource Book for Snowmass 2001’, SLAC-R-570, hep-ex/0106055-058 (four parts), May, 2001.

[7] B. A. Schumm, LCDTRK.F, http://www.slac.stanford.edu/ schumm/lcdtrk20011204.tar.gz

[8] B. C. Allanach et al., hep-ph/0202233, February, 2002.

[9] See:

http://scipp.ucsc.edu/ schumm/lctrack/national_meetings/arlington/forward_usy.ppt

[10] See http://scipp.ucsc.edu/LC

[11] C. Heusch and P. Minkowski, Phys. Lett. B374, 116 (1996).

[12] B. A. Schumm, 'Identification of a Sample of $e^{+} e^{-} \rightarrow q \bar{q}$

Events for the Precise Measurement of $\alpha_{s}$ in High-Energy Electron Positron Colliders’, hep-ex/9612013, December, 1996

[13] B. A. Schumm, A. S. Truitt, 'Update on the Measurement of $\alpha_{S}$ at a $500 \mathrm{GeV}$ Linear Collider', published in the proceedings of the $5^{\text {th }}$ International Linear Collider Workshop, Batavia, IL, October 24-28, 2000; hep-ex/0102020 (February 2001). 
[14] B. A. Schumm, 'Tracking and Vertexing at a High Energy Linear Collider', published in the proceedings of the $4^{\text {th }}$ International Workshop on Linear Colliders, Sitges, Spain, April 28 - May 5, 1999; hep-ex/9909054 (September, 1999).

[15] B. A. Schumm, 'Research and Development towards a Detector for a HighEnergy Linear Electron Positron Collider', hep-ex/0111009, published in the proceedings of the Summer 2001 Snowmass Summer Study on the Future of Particle Physics, Snowmass, Colorado, June 30 - July 21, 2001; hep-ex/0111009 (November, 2001).

[16] W. Walkowiak, 'Vertex Detection for a Charm Tag in $e^{+} e^{-} \rightarrow W^{+} W^{-}$at a High Energy Electron-Positron Linear Collider', published in the proceedings of the Summer 2001 Snowmass Summer Study on the Future of Particle Physics, Snowmass, Colorado, June 30 - July 21, 2001; hep-ex/0110039 (October 2001). 


\section{Introduction: Particle Theory at UCSC}

Theoretical particle physics research at UCSC is conducted by Professors Tom Banks, Michael Dine and Howard Haber. Banks work ranges from string theory, particularly foundational issues and questions such as the cosmological constant, to cosmology to understanding of supersymmetry and its phenomenology. Dine works on both phenomenological and foundational issues in string theory, as well as on supersymmetry, particularly issues connected with dynamical supersymmetry breaking, and cosmology. Haber is a leader in particle phenomenology, with emphasis on the theory of electroweak symmetry breaking and Higgs bosons and the phenomenology of new physics beyond the Standard Model at current and the next generation of particle colliders. The Santa Cruz theory group also profits from post-doctoral research associates, graduate students and visitors. Highlights of the theoretical research program-recent accomplishments since 2000 and future research plans - will be described below.

Santa Cruz also boasts a very active program in theoretical cosmology, under the direction of Joel Primack. Primack is a member of SCIPP, and interacts closely with the particle theorists and with experimentalists, playing an important role in advising and stimulating the growing experimental astrophysics program. He also works extensively with members of the UCSC Astronomy Department and Lick Observatory.

\section{Overview of the Research Programs of the Principal Investigators}

\section{Tom Banks}

Throughout his career, Banks has maintained a broad research program, dealing with issues ranging from phenomenology to the most basic questions in cosmology and quantum gravity. In the earlier 1980s it became clear that the fundamental problems of particle physics were unlikely to be resolved without a quantum theory of gravity. Since that time, Banks has devoted much of his time to the quantum theory of gravity, and became one of the leaders in String Theory when it was revived in 1984.

Since 1996, Banks has been working primarily on the fundamental formulation of theories of quantum gravity. He was a co-inventor of Matrix Theory, the first completely nonperturbative definition of a quantum theory of gravity. As he progressed in understanding both Matrix Theory and the formulation of Anti-deSitter gravity due to Maldacena, Banks came to realize that there were in fact many different theories of Quantum Gravity, depending on asymptotic boundary conditions. It also appeared that quantum gravity theories, which were not exactly supersymmetric, could not exist in 
asymptotically flat spacetime. Banks formulated some conjectures, relating supersymmetry breaking, and the cosmological constant, which will be described below. He also sharpened arguments for the existence of different theories of quantum gravity.

These questions are directly connected with the way in which String/M-theory can make contact with the real world. The conventional wisdom held that there was a unique theory of quantum gravity and that different realizations of it that had been discovered were analogs of different vacuum states in a single quantum field theory. This held out the hope that vacuum selection could be understood dynamically, but also led to a puzzle since there seemed to be many completely stable supersymmetric quantum ground states. Banks showed that only realizations of string theory connected along a continuous moduli space of supersymmetric vacua could be correctly understood by the field theory analogy (and even here there are subtle differences). Disconnected realizations are simply different theories, with different behavior in both the ultraviolet and the infrared (the UV and IR are connected, because high energy states are predominantly huge black holes and care about the asymptotic geometry of spacetime). Thus we have many theories of quantum gravity. The Banks-Fischler conjecture about finite numbers of quantum states in asymptotically de Sitter spaces gives us a way to understand how this degeneracy is broken. If we postulate that the correct theory of the world has a fixed number of states, we learn that it must violate Supersymmetry. This motivated Banks' further conjecture that SUSY breaking could be understood in terms of the properties of de Sitter space, leading to a gravitino mass $m_{3 / 2} \sim \Lambda^{1 / 4}$ in a space with positive cosmological constant $\Lambda$. A rough derivation of this scaling relation exists and will be described below. The hopeful claim then is that, apart from this single parameter and the Planck scale, the theory is completely fixed (at least to all orders in an expansion in powers of $\Lambda / M_{P}$. ).

Banks has also continued to work on topics of more direct phenomenological interest, mostly in collaboration with Dine. Some of this work had to do with phenomenological applications of the ideas of M-theory duality, as well as constraints from experiment on the possibility of observing M-theory phenomena like large extra dimensions, noncommutativity of spatial coordinates etc.. Other aspects were related to the uses of Mtheory moduli as inflatons or axions. In particular, Banks and Dine found models where the traditional cosmological bounds on axion decay constants could be weakened by 4-5 orders of magnitude. With Douglas we also showed that the concept of a time varying fine structure constant was problematic, within the realm of effective field theory. 


\section{Michael Dine}

A large portion of Dine's work is focused on Physics Beyond the Standard Model. He has a long-standing interest in low energy supersymmetry, the strong $\mathrm{CP}$ problem, and string theory. His work in cosmology ranges over questions of dark matter, mechanisms for baryogenesis, the moduli problem of string theory, theories of inflation, and more fundamental questions in string cosmology.

Dine's work on supersymmetry has included models of supersymmetry and supersymmetry breaking, investigations of supersymmetry dynamics and supersymmetry breaking, and issues of flavor. Much of his recent effort, as will be described below, has been directed at the question of whether low energy supersymmetry might be a string prediction, and whether string theory predicts a particular pattern of supersymmetry breaking.

On the question of strong CP violation, Dine was among those who proposed the "Invisible Axion," still among the most plausible solutions of this puzzle and the subject of active experimental search. He was among those who first appreciated the cosmological constraints on the axion, and its possible role as a dark matter particle. String theory has lead to a much more sophisticated understanding of the invisible axion, both at a fundamental level and in cosmology, and much work on this subject has been supported by the SCIPP grant during the past five years. Further work on this topic is also proposed here. Dine has also made contributions to the question of spontaneous $\mathrm{CP}$ violation as the explanation of the small value of the $\theta$ parameter of QCD, and again string theory and supersymmetry have helped shape our understanding of these subjects.

From the start of the "First String Revolution" in the mid 1980's, Dine has appreciated that string theory provides tools to investigate the full set of questions of physics beyond the Standard Model, and this has guided his investigations of string theory. Dine's research in string theory deals with both fundamental issues and issues of a more phenomenological character. Dine has throughout tried to formulate the problems of string phenomenology in a generic way, with the hope of obtaining robust predictions. As will be explained below, in recent years, he has outlined and pursued possible programs to understand whether low energy supersymmetry might be a robust prediction of string theory, and how one might obtain predictions for the pattern of soft breakings.

While, not surprisingly, the results of these efforts have been tentative, Dine has come successfully used as a testing grounds for many "beyond the Standard Model" theoretical speculations. With various collaborators, he has shown that certain forms of the soft breaking spectrum that have been widely considered are not generic outcomes of string theory. With Banks and postdocs Fox and Gorbatov, he has been able to show that a proposed mechanism for natural inflation from higher dimensional theories is not realized in String/M theory, and similarly that a class of proposals for an implementation of the anthropic principle do not have a realization in these theories. Given that String/M theory is the only sensible theory in which we can presently (and quite possibly ever) 
meaningfully investigate these questions, these results are quite striking. More studies of this sort are contemplated in this proposal.

In cosmology, in addition to axionic dark matter, Dine has worked extensively on the matter-antimatter asymmetry. His proposal with Affleck of a mechanism involving coherent scalar fields in supersymmetric theories is one of the more promising possible mechanisms, and Dine and collaborators have extensively fleshed out this scenario in the last few years. Dine also made contributions to our understanding of electroweak baryogenesis. With Banks and Graesser, Dine has recently revisited questions revolving around axions as dark matter. Dine has worked extensively on cosmological issues in string theory, particularly on the moduli problem, and on issues of cosmological backgrounds in string theory, and both recent and proposed work in these areas will be described below.

\section{Howard Haber}

The Standard Model of particle physics has been remarkably successful in describing the physics of elementary particles and their interactions up to an energy scale of a few hundred GeV. Precision measurements of electroweak observables at Fermilab, SLAC and CERN have failed to find any definitive departures from Standard Model predictions. In some cases, theoretical predictions have been checked with an accuracy of one part in a thousand or better. Some interesting deviations do exist-e.g., the NuTeV measurement of the neutral current cross sections at low energy and a possible anomaly in $g-2$ of the muon - although in both cases unresolved theoretical questions still remain which could account for the observed deviations. Nevertheless, the probability of the global Standard Model fit of the LEP electroweak working group seems to indicate a consistent picture, and favors a light weakly-coupled Higgs boson, $m_{h}<211 \mathrm{GeV}$ at 95\% CL [1].

Nevertheless, the Standard Model is not the ultimate theory of the fundamental particles and their interactions. At an energy scale above the Planck scale, $M_{\mathrm{PL}} \simeq 10^{19} \mathrm{GeV}$, quantum gravitational effects become significant and the Standard Model must be replaced by a more fundamental theory that incorporates gravity. The observation of neutrino mixing (which almost certainly implies that neutrinos are not massless) requires new physics beyond the Standard Model, although the energy scale that characterizes this new physics is likely to be quite large (typically above $10^{10} \mathrm{GeV}$ ). More theoretical arguments have been advanced to suggest that the Standard Model must break down at an energy scale near $1 \mathrm{TeV}$. far below the Planck scale. If $\Lambda$ is the scale of new physics beyond the Standard Model, then loop corrections to $m_{h}^{2}$ are expected to be of order $\left(g^{2} / 16 \pi^{2}\right) \Lambda^{2}$. Unless $\Lambda$ is below a few $\mathrm{TeV}, m_{h} \sim \mathcal{O}(100 \mathrm{GeV})$ can only be a consequence of extreme fine-tuning of the bare Higgs mass parameter. That is, the Standard Model provides no mechanism for protecting the masses of scalar fields. Assuming that the Higgs mass takes on its "natural" value, it follows that the scale of new physics $\Lambda$ cannot be much higher than a few TeV. 
A viable theoretical framework that incorporates light weakly-coupled elementary scalar fields is the theory of "low-energy" supersymmetry, where the scale of supersymmetry breaking lies no higher than $\mathcal{O}(1 \mathrm{TeV})$. Supersymmetry provides a mechanism in which scalar field masses are naturally light. In such an approach, a new class of particles, supersymmetric partners to all presently known particles, must exist with masses no larger than about $1 \mathrm{TeV}$. Such particles would be discovered at future colliders: either at the upgraded Tevatron or at the forthcoming LHC. The Higgs sector of the low-energy supersymmetric model is of particular interest because it predicts the existence of a light $\mathrm{CP}$-even neutral Higgs boson with a mass that is typically below about $135 \mathrm{GeV}$. Moreover, low-energy supersymmetric models provide a consistent framework for unification of fundamental forces and allow one to extrapolate the TeV-scale theory all the way up to the Planck scale. In particular, in the simplest models of low-energy supersymmetry, the three running gauge coupling constants of the Standard Model are unified at a very high energy scale (of order $2 \times 10^{16} \mathrm{GeV}$ ), suggestive of grand unification.

The basic themes underlying Haber's research program are: (i) the elucidation of the dynamics responsible for electroweak symmetry breaking; (ii) the viability of lowenergy supersymmetry as a model of electroweak interactions; (iii) the unification of fundamental particles and their interactions; and (iv) the search for physics beyond the Standard Model at present and future colliders. Topics of study include the following four areas of particle physics theory and phenomenology:

1. The phenomenology of the Higgs sector [2] is intimately tied to the origin of electroweak symmetry breaking. The discovery of a light CP-even Higgs boson with the properties expected in the Standard Model would be an important first step. Nevertheless, such a discovery would not be sufficient to conclude that the Standard Model has been successfully verified. Because of the decoupling properties of more general Higgs sectors $[3,4]$, the evidence for additional structure in the scalar sector will likely require precision measurements (to detect small deviations from Standard Model predictions) and higher energy experimentation to produce new scalar states.

2. The search for low-energy supersymmetry is one of the cornerstones of experimental programs at future colliders [5]. The discovery of supersymmetry would have a profound impact on theoretical particle physics and could provide our first detailed glimpse of the underlying fundamental physics at a higher energy scale. Although effective models of low-energy supersymmetry are easy to construct (the minimal supersymmetric extension of the Standard Model [MSSM] has attracted much theoretical and phenomenological attention), such models simply parameterize the effects of supersymmetry-breaking which must exist in any realistic approach. The origin of lowenergy supersymmetry breaking is not known. Although numerous approaches have been devised, no simple realistic model of supersymmetry breaking exists at present. The discovery of supersymmetry and the measurement of supersymmetric parameters could provide essential clues for the development of a theory of supersymmetry breaking. 
3. Precision electroweak physics at future colliders will continue to play a significant role. In addition to further testing the Standard Model and elucidating the scalar sector, the precision measurement of supersymmetric observables will be essential for verifying the supersymmetric interpretation of the theory and providing a deeper understanding of the model parameters.

4. In imagining what phenomena are likely to be uncovered by future collider experiments, one should not restrict ones investigations to the minimal approaches. For example, constraints on non-minimal Higgs sectors are rather mild. New phenomena in the scalar sector could provide new experimental signatures, which should not be overlooked. New sources of CP-violation often arise in theoretical approaches beyond the Standard Model. The observation of neutrino masses implies that lepton-number violation exists at some level in the low-energy effective theory (beyond the Standard Model). In the context of low-energy supersymmetric models, such phenomena can be incorporated in a number of different ways, with interesting phenomenological implications for future collider experiments.

\section{Research Programs of the Principal Investigators 2000-2003}

\subsection{Tom Banks}

I will review only the most important contributions I have made since 1999. A number of investigations carried out with M. Dine will be discussed in detail in the next section.

1. Asymptotic Darkness and the Connectivity of the space of Quantum Theories of Gravity: A paper I wrote with W.Fischler[6] emphasized that black hole production would dominate high energy scattering in a growing range of impact parameters in any theory of quantum gravity (Related ideas appeared in [7]). This supplements arguments given previously with O.Aharony and leads to the conjecture of Asymptotic Darkness: the high energy behavior of quantum gravity, and thus its fundamental formulation is described by a theory of black holes. This has enormous theoretical importance and phenomenological implications for theories of low scale quantum gravity. The qualitative signals for black hole production in such theories were first described in my paper with Fischler. In [8] I showed that these results implied that there were many disconnected theories of quantum gravity. It is incorrect to think of them all, by analogy with superselection sectors in quantum field theory, as different states of the same theory. This conclusion is reinforced by a reanalysis of vacuum tunneling that I did [9], following the classic work of Coleman and De Lucia. Finally, I showed[10] that the AdS/CFT correspondence led to the same conclusion. In particular, for negative values, the cosmological constant is a discrete tunable parameter (partially) characterizing different theories of quantum gravity. These results change many of the fundamental assumptions with which string theorists have been working since 1984 . 
2. De Sitter Space and Supersymmetry Breaking: Supersymmetry breaking is the most difficult unsolved problem in string theory. It is intimately tied with the value of the cosmological constant. Fischler and I independently suggested that the positive cosmological constant was a discrete parameter describing theories of quantum gravity with a finite number of quantum states. I further conjectured[11] that the cosmological structure of asymptotically de Sitter space led to a larger breaking of SUSY than one could understand in low energy field theory terms. In particular, in four dimensions, the gravitino mass is related (in Planck units) to the cosmological constant $\Lambda$ by the formula $m_{3 / 2} \sim \Lambda^{1 / 4}$. This predicts superpartner masses in the $\mathrm{TeV}$ range, for the value of the cosmological constant that fits cosmological data. In [12] I gave a hand waving derivation of this relation in terms of the interaction of the gravitino with a vast collection of degenerate low energy states localized on the cosmological horizon. This theory of SUSY breaking imposes strong constraints on low energy phenomenology. I gave a partial account of those constraints in [13] and will propose improvements on this analysis in my description of future work. Finally, Fischler and I made an analysis of measurement theory in de Sitter space. We showed that the phenomenon of Poincare recurrences could not be observed in a self consistent theory of a quantum de Sitter universe with a finite number of states.

3. Quantum Cosmology and the $p=\rho$ fluid: In [14,15] Fischler and I gave a prescription for quantum Big Bang cosmology based on the Fischler-SusskindBousso bound on entropy in cosmological spacetimes. The mathematical rules of this cosmology are still under construction, but we argued that it led to a picture of initial conditions near the Big Bang as a dense fluid of horizon filling black holes, with equation of state $p=\rho$. We showed that a cosmology based on these ideas solved all of the conventional problems solved by inflation (with one possible exception, see below), without an inflationary era. It also predicts a relic density of extremely heavy, highly charged magnetic monopoles, which is consistent with all current bounds, but could, in principle, be observed. The one problem with our scenario is, that if one assumes conventional field theoretic causality bounds on the range of correlations in the $p=\rho$ fluid, our model, although it gives the right power spectrum for fluctuations, cannot explain the correlations of the Cosmic Microwave Background over the long range that has been observed. Below I will describe ongoing work to remedy this defect. We also wrote a paper[16] arguing that the $p=\rho$ black hole fluid was the natural final state of a Big Crunch cosmology.

4. Cosmology of Moduli: Early in this period I also continued to work on my ongoing project of using string theory moduli to explain inflationary cosmology. Papers related to modular cosmology are [17]. 
5. Finally, I have worked on a variety of phenomenological projects, including a paper with Dine and Douglas on the time variation of the fine structure constant[18], and with Anisimov, Dine and Graesser on phenomenological bounds on noncommutative gauge theories[19].

During this period, much of my work was published only on the arXiv.org website, because I believe all other methods of publication are obsolete. I have written 24 papers since my last submission to DOE in mid 1999. Two of them have been cited over 50 times and another one over 100 times, according to information on the SPIRES website.

\subsection{Michael Dine}

During the past three years, Dine has focused on a variety of problems in supersymmetric theories, in string theories, and in cosmology. These will be described in the next three subsections.

\subsubsection{Supersymmetry, Grand Unification, and Non-Commutative Field Theory}

Dine worked on a number of phenomenological topics during this period. He was a co convener of the "TeV and Beyond" working group at Snowmass, 2001. In this role he wrote the summary talk of the String Theory subgroup, and organized a range of activities, including a panel discussion of theorists and experimentalists. With Y. Grossman and S. Thomas[20], Dine extended earlier work on slepton violation at linear colliders, one of the most exciting potential signals at a $500 \mathrm{GeV}$ facility.

In the period 2000-2003, Dine considered several problems in supersymmetric theories. With Yossi Nir and Yael Shadmi (Weizmann Institute) and his student Eric Kramer, he considered $\mathrm{CP}$ violation in supersymmetric theories[21]. This work was stimulated by early results from $\mathrm{BaBar}$ which suggested the possibility of a small value for $\sin (2 \beta)$. They noted that there is a natural framework which would predict such a result, which we dubbed "approximate CP." In this framework, CP is exact at the level of the Yukawa couplings (superpotential, in the supersymmetric action), and is spontaneously violated by dynamics associated with supersymmetry breaking. Dine and collaborators argued that this is barely compatible with the facts of the K meson system, but the value of $\frac{\epsilon^{\prime}}{\epsilon}$ is difficult to understand. They also discussed the sorts of alternative pictures which a verification of the Standard Model prediction were to be obtained, as was subsequently the case.

Following on some work by E. Witten[22], Dine, also in collaboration with Nir and Shadmi, considered a new approach to grand unification[23]. Grand unification is successful only in the context of supersymmetry. In this framework, there is the question: why are the Higgs doublets so much lighter than their triplet partners. Witten noted that in models with a certain group structure, one can understand this as a consequence of 
symmetries. Dine and collaborators argued that, in addition to this problem, there is another puzzle one should solve in these theories: the value of the grand unification scale itself. Motivated by the structure of string theories, they argued that prior to supersymmetry breaking, the value of this scale should be undetermined; correspondingly, there should be a field which breaks the gauge symmetry, with no potential. Implementing this successfully highly constrains the structure of the theory. Indeed, in subsequent work, shortly to be completed, Dine's student Kramer has shown that the gauge group and Higgs content is essentially unique. This is in contrast to the standard approach to grand unification, where there is an infinite possible variety of gauge groups and Higgs content.

Dine has investigated some phenomenological implications of non-commutative four dimensional gauge theories [19] in collaboration with graduate student Alexey Anisimov, postdoc Michael Graesser and Tom Banks. These theories have attracted some attention, in large measure because they arise in certain limits of string theory, and because of their interest to mathematicians. There has been some work on the phenomenology of these theories, setting modest limits on the scale associated with non-commutative phenomena [24] Dine et al focused on the fact that these theories necessarily violate violate Lorentz invariance, and in particular, rotational invariance. This is clearly dangerous, since the world is Lorentz invariant to a remarkably good accuracy [25]. Dine et al noted that previous analyses had considered such Lorentz violation at tree level, but that potentially much more stringent constraints arise from operators which can be generated in loops. They computed these operators in some simple models. The resulting experimental bounds on the non-commutative length scale are so stringent that it is unlikely that four-dimensional non-commutative physics will be discovered at a future collider.

\subsubsection{String Theory}

There are many obstacles to developing a string phenomenology. A number of these have been pointed out by Dine and various collaborators through the years. Among the most serious of these is the existence of a broad array of string compactifications, only a small fraction of which resemble the world we observe. Even among these, it is hard to understand how the parameters of nature could emerge as they do. At first sight, it would seem that all couplings should be either large or zero. Understanding the question of why some couplings are small and the possibly related question of why there are hierarchies between different scales is thus one of the most urgent questions in the theory.

During this period, Dine considered various possible explanations of these phenomena. One popular approach is known as the "racetrack." This is a hypothetical mechanism to obtain small gauge couplings in string theory. It is generally claimed that this 
mechanism leads to a weak coupling ground state of the theory. Dine, with Yuri Shirman, investigated this mechanism, and obtained a number of insights[26]. They showed that the assumption is not, in general correct. While the gauge coupling is small, other quantities cannot be systematically computed. They argued that this might be a general state of affairs: certain quantities might be computable, while others could not be computed by weak coupling mechanisms.

In recent years, Dine has come to the view that, while it is probably extremely difficult to find the solution of string theory which describes nature, it may be possible to extract generic predictions from string theory by examining broad classes of solutions. In particular, he has argued that it may be possible to show that string theory predicts low energy supersymmetry, and has attempted to clarify what this means. He has also argued that it might be possible to make some general statements about the pattern of supersymmetry breaking - with resulting predictions for experiments at the Tevatron and LHC colliders - without a detailed solution.

Dine has also stressed that many questions of current interest in particle physics - axions, large dimensions, the dark energy, and others - cannot be meaningfully addressed in the framework of effective field theory. Whether or not string theory is correct, as a complete, finite theory, it provides a model in which to assess the plausibility of many theoretical speculations.

One approach to predicting low energy supersymmetry would be to demonstrate the inconsistency of vacua without supersymmetry. Dine has noted that one must be careful what one means when one speaks of non-supersymmetric string vacua, and has given a definition.

Dine, with Graesser (and earlier with graduate student Josh Gray and visitor Kiwoon Choi) explored one possible type of inconsistency during the past two years: anomalies in discrete symmetries. Non-supersymmetric string vacua are typically problematic. They often possess tachyons, and even if not are frequently unstable to catastrophic decays. Banks has given arguments that they may not make sense at a non-perturbative level. One possible problem with such theories is the existence of non-perturbative anomalies. In the past, Banks and Dine[27] have argued that discrete symmetries in string theory are likely to be gauge symmetries, and that anomalies would signal an inconsistency. Dine's student MacIntire had conducted an extensive search for such anomalies in symmetric orbifold models, with supersymmetry, and found no examples (in general, anomalies do arise, but in all cases they could be cancelled by a GreenSchwarz mechanism)[28]. Dine and collaborators reasoned that perhaps one might find anomalies in non-supersymmetric strings, and that these might be generic. They first noted, following earlier work of Berkooz et al[29], that such conditions do exist in Type I theories (both with and without supersymmetry), and extended slightly the range of models for which this is an issue. They then examined a variety of symmetric orbifold models of the heterotic string theory, without supersymmetry. Again, they found that anomalies could be cancelled. They then studied asymmetric orbifolds. In addition to 
implementing existing technology for studying the spectra of these models, they had to face a number of issues, such as determining the ground state charges in twisted sectors. They found, in the full range of models studied, cancellation of anomalies. Witten has observed that in $G_{2}$ models, such a cancellation also occurs[22]. Dine and Graesser are preparing a paper which reviews the general issues and discusses specific examples. As a biproduct of these investigations, they have shown that certain constraints argued to hold for discrete symmetries are not true, in general. They will also discuss the question of CPT conservation in string/M theory.

While this investigation has taught us a good deal about aspects of discrete symmetries in string theory, it has not succeeded in distinguishing supersymmetric from non-supersymmetric theories. Another phenomenon which distinguishes supersymmetric and non-supersymmetric theories was pointed out long ago by Witten[30]. He noted that even in the simplest Kaluza-Klein theories, there is an instability which leads to a decay to - literally - nothing. This occurs, however, only in non-supersymmetric theories. The meaning of this decay is obscure. It is also not clear whether it is generic to non-supersymmetric theories, though some generalizations are known[31]. Dine is currently investigating this phenomenon, in collaboration with UCSC post-docs Gorbatov and Fox. This will be described further under "current and proposed research." Dine remains optimistic, as does Banks, that a sharp statement may be possible.

It is often remarked (in fact Dine was one of the first to do this counting) that if supersymmetry is discovered, there will be (at least) 105 new parameters to measure. While at first sight this may seem discouraging, the absence of flavor changing neutral currents for light quarks strongly suggests that the soft breakings will exhibit certain striking regularities; these regularities are likely to provide important clues to the mechanisms of supersymmetry breaking.

Perhaps the simplest proposal to resolve these flavor puzzles is known as "gauge mediation"[32], put forth by Dine and Nelson. More recently, Dine has argued that gauge mediation is a plausible outcome of string theory. In particular, one of the great puzzles of string theory is the question of how the underlying parameters ("moduli") are fixed. He has suggested that these parameters are likely to be fixed at a point with a high degree of symmetry, in which case gauge mediation is an almost inevitable outcome. This proposal also resolves one of the most serious cosmological problems of string theory[33]. Only a few other viable proposals have been put forward for these regularities, and Dine has stressed (e.g. in his contribution to the 2002 Snowmass Proceedings) that all of them are either motivated by string theory or have a realization in string theory.

While it is difficult to say what string theory predicts for various phenomena, one outcome of Dine's researches in this period has been the realization that one can often state what string theory does not predict. One example involves one of these proposals to explain the squark and slepton spectrum: anomaly mediation[34,35]. Anomaly mediation (and its cousin, gaugino mediation[36]) makes definite predictions for the soft breaking spectrum. It has two ingredients: the first is the existence of certain anomalous 
contributions to gaugino (first noted by Dine and MacIntire[37], and fully understood more recently[34,35]) and squark masses. The second is a particular form for the Kahler potential[35]. Randall and Sundrum argued that this "sequestered" form arises naturally in a brane world picture, in which supersymmetry is broken by interactions on one brane, while the fields of the Standard Model propagate on a distant brane. While it is difficult to determine the true vacuum of string theory, there are many situations where it is possible to calculate the Kahler potential, and it is possible to test the hypothesis that separated branes lead to this special form for the Kahler potential. Dine, in collaboration with his student, Anisimov, postdoc Michael Graesser, and Scott Thomas (Stanford), investigated a range of brane world set ups in string/M theory, and found that, in no case which can be analyzed in a systematic approximation, does a sequestered Kahler potential emerge[38]. They noted that there are some very special situations, where calculations are not currently possible, which might yield such a potential, but evidence from the calculable examples raises serious questions about these as well.

Other such "non-predictions" of string theory discovered by Dine and collaborators during this period are discussed in the section on cosmology.

Dine has also considered other issues in string theory, without direct phenomenological implications. Recently, for example, he has completed an investigation (with Gorbatov, as well as with I. Klebanov of Princeton University and his student M. Krasnitz) of certain tachyons in closed string theories[39]. In recent years, following suggestions of Sen[40], there has been significant progress in understanding tachyons in open string theories. For the most part, however, the significance of tachyons in closed string theories has remained obscure. Dine and collaborators noted that there are certain instances in which one can compute the tachyon potential, however, determining, for example, whether or not a minimum exists. The analysis is quite simple and clarifies a number of issues related to these fields.

\subsubsection{Cosmology}

During this period, Dine maintained his longstanding interest in the question of baryogenesis. "Affleck-Dine baryogenesis" is a process for generating the baryon asymmetry in supersymmetric theories, through the classical motion of scalar fields. A few years ago, Dine, with Lisa Randall and Scott Thomas, investigated many aspects of this process[41]. The mechanism depends crucially on the existence of flat directions in the scalar potential, and its efficacy is a function of the degree to which the potential is indeed flat. Recently, Campbell and Ellis picked up on one of the issues raised in this work[42]. Dine et al had noted that in the case that the relevant flat directions were not particularly flat, thermal effects could be very important, and were likely to prevent baryogenesis all together. Campbell and Ellis argued that this analysis had neglected the effect of thermal masses, and that these could lead to an adequate baryon asymmetry. As a result, Dine and his student A. Anisimov[43] conducted a more thorough analysis 
of thermal effects. They found that one of the principle effects discussed by Campbell and Ellis (the generation of "thermal A terms") does not occur, in general, because of symmetries. They noted, however, that there are a variety of other effects which can result in a suitable asymmetry. Even in the case of rather flat directions, there are thermal effects which can appreciably alter the predicted asymmetry. Anisimov undertook a more thorough investigation of this problem[44].

In addition, Dine has just completed an article for Reviews of Modern Physics on Baryogenesis with A. Kusenko[45].

With Banks and postdoctoral fellow Michael Graesser, Dine reconsidered the cosmological limit on the axion decay constant (mass)[46]. They noted that the usual limit assumes a very simple evolution of the early universe. However, in supersymmetric theories, the axion has a scalar partner, often called the saxion, whose cosmological problems are parametrically much more severe than those of the usual axion. They argued that it does not make sense to discuss limits on the axion unless one has solved these other problems. They reviewed the various proposed solutions to theses difficulties, and found that typically the bounds on the axion are significantly relaxed. This is possibly disappointing, in that it might mean that the axion is the dark matter, but cannot be observed in current axion searches (models in which the axion is detectable were described in this paper). However, it is also interesting, because the axions predicted by string theory (which are, in many respects, the most compelling axion models) often naturally have decay constants in the allowed range.

Some of Dine's work on cosmology during this period has already been alluded to in the previous section. In particular, Dine proposed a solution to the moduli problem of string theory, the problem that the moduli fields typically carry far too much energy in the early universe. The proposal involves the realization (developed earlier in work with L. Randall and S. Thomas[47]), that if the minimum of the moduli potential lies at a point of enhanced symmetry, the system can easily end up there during a period of inflation. In [33], Dine fleshed out this proposal in more detail, connecting it to earlier observations about the racetrack mechanism and other suggestions for supersymmetry breaking. Dine also discussed solutions of the "Brustein-Steinhardt" problem, which arises in this context. Related work was done by Ovrut and Steinhardt[49].

Dine also worked on more fundamental questions in string cosmology during this period. With Banks, as described above, he considered a number of questions in string cosmology. Banks and Dine[48] described a simple string cosmology, in which, both in the distant past and future, one might hope that a weak coupling description is applicable. The difficulties both near a big crunch and a big bang were considered. Further work along these lines is in progress. In this work, Banks and Dine also made a number of other observations. For example, they pointed out that the QCD axion might provide not only the dark matter, but could also explain the dark energy as well. 
Dine and collaborators also considered phenomena which do not seem to be predicted by string theory. One of these has immediate experimental/observational implications. In collaboration with Banks and Michael Douglas of Rutgers University, examined the question, raised by recent studies of quasar absorption lines, of time variation of fundamental constants[18]. They noted that within the framework of effective field theory, a field with the properties required to explain the data is implausible (similar observations have been made by Donoghue[50]). These analyses require a highly plausible assumption about cutoffs. One can construct field theory models where the issue can be addressed in a finite computation, which support this conclusion. Banks, Dine and Douglas also showed that in string theory, where the role of the cutoff is played by the string scale, these conclusions also hold. As noted above, this means that if the observations (and their interpretation) of variable $\alpha$ are confirmed, they will challenge some of our most basic notions in physics.

Dine considered other hypotheses for exotic physics which have been put forward for various reasons which can be addressed in string theory. In his summary talk at the conference at the Space Telescope Science Institute on Dark Matter and Dark Energy[51], he discussed a suggestion of Vilenkin's to solve the cosmological constant problem anthropically[52]. This proposal requires the existence of extraordinarily light fields, with peculiar properties. The plausibility of such a field cannot be assessed without an underlying fundamental theory, such as string theory. Dine noted that a preliminary investigation indicated that such fields do not arise in string theory, in any regime where one can calculate. More generally, Dine has argued that the question: does string theory provide a setting for the anthropic principle? is a scientific one[51]. Recent work by Lisa Randall and Nima Arkani-Hamed[53] have added urgency to this question, which is currently under investigation by Banks, Dine, Gorbatov and Fox. This will be described briefly in the next section. Briefly stated, however, it appears that under further investigation, these earlier, preliminary conclusions hold.

In earlier work with Banks and Motl[54], Banks, Dine and Motl investigated another proposed anthropic solution[55] to the cosmological constant problem. They pointed out a number of difficulties with such a solution. Recent work by a group at Stanford has sharpened some of these proposals[56], and Banks, Dine, Fox and Gorbatov have launched a study of these issues.

\subsection{Howard Haber}

From 2000-2003, I completed a report on the Higgs physics program for the upgraded Tevatron, served as a co-convener of the Electroweak Symmetry Breaking (P1) Working Group at the 2001 APS/DPF/DPB Summer Study on the Future of Particle Physics (Snowmass 2001), and co-organized the Higgs Working group of the American Linear Collider Collaboration. My research focused primarily on the following topics: (i) the theory and phenomenology of Higgs bosons in the decoupling limit; (ii) a program of 
precision Higgs measurements at the linear collider (LC); (iii) lepton-number-violating supersymmetry; (iv) Higgs contributions to the muon $g-2$; and (v) CP-violating observables in low-energy supersymmetry. Work on this last topic is not yet complete and will be briefly discussed in the section on ongoing and proposed research.

\subsubsection{The Higgs Boson Discovery Reach of the Upgraded Tevatron}

Run-2 of the Tevatron collider was initiated in 2001 after a significant upgrade of the Tevatron accelerator. Prior to the completion of the upgrade, the Fermilab Theoretical Physics Department (in association with the two collider experiments CDF and D $\varnothing$ ) sponsored a series of workshops to assess the full potential of the Tevatron Run-2. The first of these workshops focused on Higgs physics and low-energy supersymmetry. The goals of this workshop were twofold. The first goal was to organize and improve our understanding of all possible variants of weak-scale supersymmetry which may manifest themselves in collider experiments at Tevatron Run-2. The second goal was to explore the theoretical upper bounds on the mass of the lightest MSSM Higgs boson (given a variety of both strong and weak theoretical assumptions), improve our understanding of multi-channel Higgs searches at the Tevatron, and refine the discovery reach estimates for integrated luminosities in the range $2-30 \mathrm{fb}^{-1}$.

I served as a co-convener for the Higgs working group along with Marcela Carena, John Conway and John Hobbs. Our working group examined Higgs search strategies at Run-2 of the Tevatron, with particular attention to the search for the Standard Model Higgs boson, the Higgs bosons of the MSSM, and possible extensions thereof. The MSSM Higgs sector is of particular interest to the Tevatron search, since the mass of the lightest Higgs boson is predicted to lie below about $135 \mathrm{GeV}$ [57]. The Higgs discovery potential for a further upgraded higher luminosity Tevatron (with an integrated luminosity from 10 to $30 \mathrm{fb}^{-1}$ ) was also examined. The report of our working group findings was completed in October, 2000 [58].

Three crucial Higgs mass ranges were identified: (i) $115 \mathrm{GeV} \lesssim m_{h_{\mathrm{SM}}^{0}} \lesssim 135 \mathrm{GeV}$; (ii) $135 \mathrm{GeV} \lesssim m_{h_{\mathrm{SM}}^{0}} \lesssim 180 \mathrm{GeV}$; (iii) $180 \mathrm{GeV} \lesssim m_{h_{\mathrm{SM}}^{0}} \lesssim 210 \mathrm{GeV}$. These regions arise due to the following considerations. First, the Higgs search at the LEP collider showed no definitive evidence for the production of a Standard Model Higgs boson, and concluded that $m_{h_{\mathrm{SM}}^{0}} \geq 114.4 \mathrm{GeV}$ at $95 \% \mathrm{CL}$ [59]. Second, the Standard Model global fit to electroweak data of the LEP Electroweak Working Group requires a Higgs boson with $m_{h_{\mathrm{SM}}^{0}} \leq 211 \mathrm{GeV}$ at $95 \% \mathrm{CL}$ [1]. Finally, in the minimal supersymmetric extension of the Standard Model (MSSM), the lightest CP-even Higgs mass is bounded from above- the predicted bound of $135 \mathrm{GeV}$ arises assuming that all supersymmetric masses are no larger than a few $\mathrm{TeV}$ [57].

With the three mass ranges noted above, the Higgs working group examined numerous Higgs search strategies, both in the context of the Standard Model and the MSSM. In Run- 2 with $2 \mathrm{fb}^{-1}$ delivered by the Tevatron to each detector, the Standard Model 
and MSSM Higgs boson discovery reaches cannot be improved from the current LEP limits. However, dramatic improvements can be made by extending Run- 2 in order to eventually accumulate $15-30 \mathrm{fb}^{-1}$ of data. With such a data set in hand (while maintaining both the $\mathrm{CDF}$ and $\mathrm{D} \varnothing$ detectors in order to effectively double the total integrated luminosity), the Higgs discovery reach can be significantly extended beyond the final LEP limits. For the Standard Model Higgs boson, a $3 \sigma$ discovery signal could be made for Higgs masses all the way up to $180 \mathrm{GeV}$. Moreover, with $15 \mathrm{fb}^{-1}$ of data, it will be possible to achieve a $5 \sigma$ Higgs discovery if $m_{h_{\mathrm{SM}}^{0}} \simeq 115 \mathrm{GeV}$, a value just above the present LEP limit. These results also suggest that there would be a significant fraction of the MSSM Higgs parameter space accessible to an upgraded Tevatron. Indeed, the results of our analysis showed that with luminosities above $10 \mathrm{fb}^{-1}$, nearly all of the MSSM Higgs parameter space could be ruled out at 95\% CL, a substantial improvement over the final LEP limits. The MSSM Higgs discovery reach is also improved beyond that of LEP, although one would need $30 \mathrm{fb}^{-1}$ of data (times two for the two detectors), to ensure discovery of the MSSM Higgs boson in all but a few peculiar corners of the parameter space. In addition, in one interesting region of MSSM parameter space (at large $\tan \beta$ ), the associated production of a Higgs boson and a $b \bar{b}$ pair is significantly enhanced and provides the potential for a Higgs discovery at Run-2 luminosities.

The potential for such an important discovery presents a compelling case for an extended Run-2 at an upgraded Tevatron prior to the start of the LHC. Such a run would also significantly extend the searches for supersymmetric particles and other signals of new physics beyond the Standard Model associated with the electroweak symmetry breaking scale. Although the upgraded Tevatron has been slow to reach its design luminosity of $2 \mathrm{fb}^{-1}$ per year (it is presently about a factor of three short of this goal), the Fermilab management hopes to continue to improve the Tevatron performance and eventually exceed its initial design goal with further upgrades. A total data set of $10 \mathrm{fb}^{-1}$ before the start of LHC is still possible and would provide significant opportunities for the Higgs physics program at Fermilab.

\subsubsection{Electroweak Symmetry Breaking at Future Colliders}

With the LEP program completed, the upgraded Tevatron facility up and running, and the LHC construction well underway, the DPF organized a a major three week workshop in Snowmass, CO in June, 2001 to address the long range future for US particle physics. This gathering encompassed particle theorists and experimentalists, detector designers, machine builders, accelerator physicists, astroparticle physicists and string theorists. One aim of the workshop was to contribute to the twenty year road map for the future of particle physics that was being developed by HEPAP.

I served as a co-convener of the Electroweak Symmetry Breaking (P1) working group at the 2001 Snowmass meeting. Our executive summary [60] reviewed the main candidates for theories of electroweak symmetry breaking and discussed the criteria for 
distinguishing among the different approaches. The potential for observing electroweak symmetry breaking phenomena at future facilities was outlined, with particular emphasis of the importance of a high-luminosity $e^{+} e^{-}$linear collider for clarifying the underlying electroweak symmetry breaking dynamics.

In addition, my activities at Snowmass included an effort to organize a consensus among the physics working groups for the future direction of US particle physics. In our consensus document, we argued that deciphering the mechanism that breaks the electroweak symmetry and generates the masses of the known fundamental particles is one of the central problems of particle physics. We expect the LHC to provide the initial discovery of electroweak symmetry breaking dynamics (although there is still a window of opportunity for the Tevatron as noted above). Nevertheless, to fully unravel the details of the mechanism responsible for electroweak symmetry breaking, a future collider capable of precision measurements will be necessary. A very high energy $e^{+} e^{-}$Linear Collider (LC) with an energy of $500 \mathrm{GeV}$ (with a possible upgrade to $1 \mathrm{TeV}$ ) provides a very compelling facility at which the physics of electroweak symmetry breaking and the associated dynamics of possible new physics phenomena beyond the Standard Model can be studied in great detail.

I am also currently serving as a co-convener of the Higgs Working Group of the American Linear Collider Collaboration (ALCC). In a report prepared for the 2001 Snowmass workshop, the ALCC drafted a document outlining the detailed physics case for the LC as the next forefront high-energy collider after the LHC. I was one of the authors responsible for the chapter on Higgs physics in this report. This latter work was recently expanded and updated by John Gunion, Rick van Kooten, and me, and will appear in a book that will be published later this year [61].

\subsubsection{Higgs theory and phenomenology in the decoupling limit}

Suppose that electroweak symmetry breaking is generated by the dynamics of a weaklycoupled Higgs sector. How will we determine if the Higgs sector is that of the minimal Standard Model, or if the Higgs sector is non-minimal? First, consider the Standard Model Higgs boson. At tree-level, the Higgs self-coupling is related to its mass. If $\lambda$ is the quartic Higgs self-interaction strength, then $\lambda=3 m_{h_{\mathrm{SM}}^{0}}^{2} / v^{2}$ (where $v \simeq 246 \mathrm{GeV}$ is the Higgs vacuum expectation value which is fixed by the $W^{ \pm}$mass: $v=2 m_{W} / g$ ). This means that one cannot take $m_{h_{\mathrm{SM}}^{0}}$ arbitrarily large without the attendant growth in $\lambda$. That is, the heavy Higgs limit in the Standard Model is non-decoupling. In models of a non-minimal Higgs sector, it is sometimes not possible to take any Higgs mass much larger than $\mathcal{O}(v)$ without finding at least one strong Higgs self-coupling [3]. However, in models such as the MSSM, one finds that the non-minimal Higgs boson masses can be taken large at fixed Higgs self-couplings. Since the Higgs self-couplings in the MSSM are gauge couplings (and thus are always $\lesssim \mathcal{O}(1)$ ), the heavy Higgs states decouple as the mass of the CP-odd Higgs boson $(A)$ grows large (i.e., for $m_{A^{0}} \gg m_{Z}$ ), while both 
light and heavy Higgs bosons remain weakly-coupled. In this decoupling limit, exactly one neutral CP-even Higgs scalar remains light, and its properties are precisely those of the (weakly-coupled) Standard Model (SM) Higgs boson.

In the MSSM, the deviations of the light Higgs boson properties from those of the SM reflect both the properties of the heavier Higgs states and the supersymmetric particle spectrum that enters into Higgs phenomenology via radiative corrections. Certain supersymmetric radiative corrections can be enhanced by factors of the ratio of the two neutral Higgs vacuum expectation values, $\tan \beta$, leading to an interesting pattern of deviations of the light $\mathrm{CP}$-even Higgs couplings relative to those of the Standard Model. Marcela Carena, Heather Logan, Steve Mrenna and I have studied the theoretical predictions for the CP-even Higgs branching ratios and widths as a function of the supersymmetric parameters near the decoupling limit [62]. We applied this theoretical analysis to the precision study of Higgs boson decays at a future linear collider (LC). By comparing our results with Monte Carlo estimates for the anticipated accuracy of Higgs branching ratio measurements at the $\mathrm{LC}$, we are able to deduce the ultimate discovery reach of the LC as a function of the MSSM and Higgs sector parameters. In particular, since exact decoupling occurs in the limit of $m_{A} \gg m_{Z}$, the absence of a measurable deviation from SM Higgs predictions would in general set a lower bound on the value of $m_{A}$ (which can depend somewhat on the other MSSM parameters). However, we noted the existence of an interesting phenomenon in which a decoupling-like behavior sets in for special values of the MSSM parameters independent of the value of $m_{A}$. Thus, in these special regions of the parameter space, sensitivity to $m_{A^{0}}$ is lost if no deviation from SM-like behavior of the light CP-even Higgs boson is observed.

In some cases, decoupling can be delayed. It has also been known for some time that significant radiative corrections modify the tree-level predictions of the partial width of the lightest CP-even Higgs boson, $h^{0} \rightarrow b \bar{b}$. This is the dominant Higgs decay in the mass range of interest for LEP and the Tevatron. Peculiar regions of the MSSM parameter space were identified in which the branching ratio for $h^{0} \rightarrow b \bar{b}$ is significantly suppressed. In [63], we re-examined the theoretical computation of this decay rate. We were particularly interested in two questions. First, how reliable is the theoretical calculation in regions where the branching ratio is significantly suppressed? (Such regions are clearly important for the Higgs searches at the Tevatron which depend on a substantial $b \bar{b}$ Higgs branching ratio.) Second, how is the decoupling region approached? Clearly, decoupling must occur when $m_{A^{0}} \gg m_{Z}$. In this case, one finds the following interesting behavior. Decoupling is delayed when $\tan \beta \gg 1$, since the decoupling corrections behave as $m_{Z}^{2} \tan \beta / m_{A^{0}}^{2}$. Moreover, at fixed $m_{A^{0}}$, nontrivial corrections remain even in the parameter region in which the supersymmetric mass parameters are very large. In [63], we provided explicit results for the supersymmetric QCD corrections to $h^{0} \rightarrow b \bar{b}$ (along with a number of useful analytic approximations), and explored in detail the approach to the decoupling limit as a function of the relevant supersymmetric particle masses. 
Of course, the decoupling limit is more general than the MSSM Higgs sector. For example, it is easy to construct models of extended Higgs sectors where the mass scale characteristic of the Higgs scalars beyond the SM lies somewhat above the scale of electroweak physics (the separation of scales need not be too large). In that case, it is possible to argue that the properties of the lightest $\mathrm{CP}$-even scalar will strongly resemble those of the SM Higgs boson. If the heavier states cannot be produced at a future collider, then one must rely on precision measurements of the light CP-even scalar properties in order to distinguish the SM Higgs sector from an extended Higgs sector.

John Gunion and I have examined the circumstances in which a light Higgs boson can have SM-like branching ratios and production rates and their relation to the decoupling limit [4]. We have derived expressions for the Higgs masses as a function of the Higgs self-couplings and vice versa, providing a complete translation table between the different parameter sets that can be used to characterize the model. We have carefully evaluated (in a model-independent way) the deviation of the light Higgs properties (couplings to fermions, gauge bosons and self-couplings) from those of the SM Higgs boson as the decoupling limit is approached. We expect that this analysis will provide useful tools for Higgs searches at future colliders. Our initial work assumes that the Higgs sector is CP-conserving. However, one can also consider the most general Higgs potential, which yields $\mathrm{CP}$-violating Higgs interactions. We can define a decoupling limit in the general CP-violating model, in which $m_{H^{ \pm}} \gg m_{W}$. One consequence of this limit is that the properties of the lightest neutral Higgs state again approaches those of the SM Higgs boson. In particular, the lightest neutral state is CP-even, with CP-violating couplings suppressed by a factor proportional to $m_{W}^{2} / m_{H^{ \pm}}^{2}$. (The heavier neutral states do not possess a definite $\mathrm{CP}$ quantum number.) Likewise, when the couplings to three generations of quarks is considered, the Higgs-mediated flavor changing neutral currents expected in the most general model are suppressed in the decoupling limit and could provide an explanation to the experimental absence of such effects.

\subsubsection{Lepton-number Violating Low-Energy Supersymmetry}

The observation of neutrino mixing phenomena implies that the Standard Model (which by definition incorporates massless neutrinos) must be extended [64]. In [65], Yuval Grossman and I noted that in any supersymmetric model with nonzero Majorana neutrino masses, the sneutrino and antisneutrino mix. One possible choice is to consider the supersymmetric extension of the seesaw model. A second possibility, which does not require the addition of new fields beyond those of the MSSM is the R-parity-violating (RPV) extension of the MSSM with a conserved baryon number B. Such models provide a viable framework for particle physics with lepton number violating interactions, while guaranteeing proton stability in the low-energy theory. Grossman and I have explored the general structure of neutrino and sneutrino masses in the RPV MSSM in [66,67]. 
Many authors have noted that in R-parity-violating supersymmetry, only one neutrino mass is generated at tree-level (as long as the neutral scalar vacuum expectation value is not aligned with the generalized $\mu$-parameter of the model). The two other neutrinos gain mass via one-loop radiative corrections. There are a number of mechanisms for generating the radiative neutrino masses. Most papers in the literature focus on one-loop contributions that arise from R-parity-violating Yukawa couplings. However, Grossman and I have argued [68] that these corrections are not likely to be the dominant contributions to neutrino masses. In fact, in most models, it is the sneutrinoantisneutrino mass splitting (which arises from a generalized soft-supersymmetry-breaking $B$-term) which generates the dominant contribution to the radiative neutrino masses.

The detailed phenomenology of neutrino masses and sneutrino mass-splitting depends on numerous soft-supersymmetry-breaking parameters in the low-energy effective theory. The fundamental origin of these parameters is unknown. However, one can imagine various scenarios in which these parameters are generated at some high energy scale with specific patterns (due to symmetries that are imposed on the theory at the high energy scale). Then one would use renormalization group evolution to determine the corresponding values of the low-energy parameters.

In order to perform such an analysis, it is important to study the R-parity-violating effects in a basis-independent manner. For example, in R-parity-violating theories, the "down-type" Higgs superfield cannot be distinguished from the lepton superfield (since they possess the same gauge quantum numbers). One often defines the Higgs direction to lie along the direction of the "down-type" Higgs vacuum expectation value. But, this is a basis choice. In particular, it is not an invariant direction under renormalization-group running. Thus, the first step in our program is to provide a completely basis-independent analysis of the neutrino/neutralino and sneutrino/Higgs boson sectors of the R-parityviolating model. We have succeeded in deriving expressions for neutrino masses and sneutrino squared-mass splittings in completely basis-independent formalism [67]. We hope to exploit these results in a renormalization-group (RG) analysis and make contact between various model assumptions at high-energy and the phenomenology of the lowenergy effective theory.

A second application of the basis-independent formalism was to clarify the distinction between spontaneous and explicit R-parity-breaking. Naively, one associates spontaneous R-parity-breaking with the existence of a sneutrino vacuum expectation value, although this is clearly a basis-dependent characterization. A theory of spontaneous Rparity-breaking would possess a massless Goldstone boson-the majoron. In a model with only Higgs doublets, the existence of a massless majoron is excluded, since otherwise it would have been observed (or deduced) from precision studies of $Z$ decays. In ref. [69], we formulate a basis independent condition that can test for the presence or absence of a majoron in R-parity-violating supersymmetric models. In addition, we obtained a formulae for the mass of the would-be majoron in the presence of explicit 
R-parity-violating effects. In this case, we can identify the would-be majoron as a sneutrino, and we are able to understand how its mass can be of order the electroweak breaking scale even if explicit R-parity-breaking effects are small.

\subsubsection{New physics contributions to $(g-2)_{\mu}$}

Recent data from Brookhaven reports a deviation of the measured value of $g-2$ of the muon from its Standard Model (SM) prediction [70]. There are four possible interpretations of this discrepancy: (i) a statistical fluctuation; (ii) an incorrect experimental observation; (iii) an error in the theoretical Standard Model computation, which would most likely due to systematic uncertainties associated with the evaluation of the hadronic vacuum polarization; or (iv) new physics beyond the Standard Model which contributes to $(g-2)_{\mu}$ consistent with the observed deviation. The correct interpretation is far from clear. In particular, the theoretical analysis of the hadron vacuum polarization is plagued by an apparent inconsistency of low-energy $e^{+} e^{-} \rightarrow$ hadrons data and $\tau \rightarrow \nu_{\tau}+$ hadrons data.

Nevertheless, it is useful to survey various possible theories of new physics to assess the possibility of a successful explanation for the observed $(g-2)_{\mu}$. One of the simplest extensions of the Standard Model consists of enlarging the Higgs sector. The two-Higgs doublet model (2HDM) with flavor conserving couplings arises in a number of models of new physics (including models of low-energy supersymmetry). Athanasios Dedes and I examined the contribution of $(g-2)_{\mu}$ due to Higgs exchange [71]. We concluded that a light Higgs boson in the framework of a CP-conserving two-Higgs-doublet model with flavor-conserving couplings can be responsible for the deviation observed by the BNL experiment only if $m_{\Upsilon} \lesssim m_{h} \lesssim 2 m_{B}, \tan \beta \sim 30$ and the $h Z Z$ coupling is significantly suppressed ( $\lesssim 0.06 \%$ of the SM $h Z Z$ coupling). These requirements are necessary in order to avoid the constraints from CESR $(\Upsilon \rightarrow h \gamma)$ and LEP $\left(e^{+} e^{-} \rightarrow h f \bar{f}, f=b\right.$ or $\tau)$. In addition, the other Higgs bosons of the $2 \operatorname{HDM}\left(H, A\right.$ and $\left.H^{ \pm}\right)$should be heavy enough to be consistent with the non-observation of $H Z, h A$ and $H^{+} H^{+}$production at LEP.

Our results were based on a one-loop calculation. The authors of Ref. [72] have argued that significant two-loop contributions contribute to $(g-2)_{\mu}$ due primarily to the effect of one particular [Barr-Zee] diagram, which is of the same order as the corresponding one-loop contribution, but with opposite sign. This implies that $m_{h} \lesssim m_{b}$ in order that the total Higgs contribution to $(g-2)_{\mu}$ lie within the range suggested by the BNL measurement. However, this possibility is excluded by the non-observation of $\Upsilon \rightarrow h \gamma$ at CESR. Ref. [73] argues that the two-loop contribution of the Barr-Zee diagram could change the sign of the one-loop Higgs contribution, in which case a light CP-odd Higgs boson could generate an overall positive contribution to $(g-2)_{\mu}$ of the required magnitude. However, there is at present no complete two-loop computation of 
the electroweak contribution to $(g-2)_{\mu}$ in the 2HDM. Whether the dominance of the Barr-Zee diagram survives a more complete two-loop analysis remains to be seen.

\section{Research Programs of the Post-Doctoral Research Associates}

\subsection{Michael Graesser}

Graesser was a research associate at UCSC during much of this period. He conducted several investigations with Michael Dine, which have been described in the previous section. Some of theses projects are continuing, particularly a study of CPT in string theory. This is motivated, in part, by a desire to understand the proposal of Lykken and collaborators[74]

During this period, Graesser also worked on problems associated with the RandallSundrum model, looking particularly at questions associated with radion stabilization. Graesser also studied, with Thomas, the possible significance of any discrepancy in the muon $g-2$. Of course, at the present time, one cannot say with confidence that there is or is not a discrepancy. Graesser and Thomas discussed how the size of the muon dipole moment required to explain the muon $g-2$ measurement could, under certain assumptions of flavor universality and proportionality, be used to set the overall scale for other leptonic dipole operators [75]. For instance, the magnitude of the muon dipole operator together with the current bound on the electron dipole operator can be used to bound the phase of the electric dipole operator. Similarly, the bound on flavor-violating decays could be used to limit the fractional mixing in the selectron-smuon mass matrix.

\subsection{Patrick Fox}

Kaluza and Klein demonstrated how a 5D theory gravity in a space with one compact direction looks at low energies like a 4D theory of gravity and electromagnetism. Witten [30] investigated the stability of this Kaluza-Klein spacetime and discovered that it is unstable to the formation and growth of the "Witten bubble". This is a catastrophic event from the point of view of the 4D observer, a bubble of "nothing" forms in the middle of his spacetime and then grows at the speed of light. Earlier Coleman and DeLuccia [76] investigated vacuum decay by the formation of a bubble of true vacuum in the false vacuum. With Dine and Gorbatov, Fox has redescribed Witten's bubble as a Coleman-Deluccia type problem with peculiar boundary conditions.

Embedding these theories in string theory results in a potential being generated for the radius modulus. Fox and collaborators consider the Witten bubble in the presence of this potential. They find that depending on the details of the potential the Kaluza-Klein space can still decay through the Witten bubble.

In the standard picture of big bang cosmology there is a period in the evolution of the universe when it undergoes rapid expansion. During this period of exponential growth, 
commonly called inflation, the universe grows in size by at least a factor of $e^{60}$. Inflation solves many problems of big bang cosmology, such as the flatness and homogeneity of the universe on large scales and the low density of cosmological relics. One model of inflation is chaotic inflation, which uses the slow roll of a scalar field, the inflaton, to drive a period of inflation. In order to get sufficient e-foldings the potential must be very flat and the field must roll a long distance in field space. Recently there has been interest in natural inflation where the inflaton is a pseudo-Goldstone boson [53], described by an angular variable. In order for this to be viable the periodic field must have a decay constant, $f$, larger than the 4D Planck scale, $M_{p}$.

String theory is the only known theory in which it makes sense to discuss the possibility of decay constants this large. In string theories there are many periodic variables which could play the role of the natural inflaton, for example higher rank tensor fields compactified on some internal manifold. With Banks, Dine, and Gorbatov, Fox conducted a survey of many string vacua and searched for viable periodic moduli. He found that, in all cases over which we had control, there were no inflaton candidates whose decay constant is larger than the 4D Planck scale. For theories with enough supersymmetry, extreme regions of moduli space, shrinking radii or large couplings, can always be mapped to weak coupling large radii regions of another string theory [79]. In these regions $f$ is always smaller than $M_{p}$. In cases with smaller amounts of supersymmetry he found that instanton effects became important in the regimes necessary for a large $f$. There are contributions from all instanton sectors up to $n \sim f / M_{p}$, causing the inflaton potential to vary on scales smaller than $f$. When the inflaton is rescaled such that the potential again varies on scales $f$, the decay constant has been rescaled to $M_{p}$. It would be very interesting to elevate our difficulties to the level of a no-go theorem, or to find an example where there is a field with a large decay constant in string theory. This is the subject of continuing investigation.

\subsection{Elie Gorbatov}

Non-supersymmetric String Theory vacua remain poorly understood compared to their supersymmetric analogs. Most of the non-supersymmetric string theories contain tachyons. The physical nature of open string tachyons has been investigated and more or less understood in the last few years. The fate of the closed string tachyons is a lot more mysterious.In collaboration with M. Dine, I. Klebanov, and M. Krasnitz Gorbatov investigated some dynamics of the closed string tachyon in the Rhom compactification of TypeII superstring.In particular we computed the quartic contribution to the effective action. He found that the quartic term is positive and discussed some redundancies in its definition. He then studied the evolution of this unstable system and concluded that it is typically driven to a strong coupling where the computation is no longer under control.

In a related project with Dine and Fox (described at greater length in Fox's section), 
Gorbatov is considering issues connected with the decay of the Kaluza-Klein vacuum discovered by Witten.

In another project in collaboration with T. Banks, M. Dine, and P. Fox, Gorbatov is investigating the possibility of realizing large axion decay constants in string theory. The problem is of much interest since such decay constants lead to flat potentials for the axion and so could serve as a model for inflation. Our efforts focused on trying to understand whether we could find the periodic moduli fields in string theory compactifications that naturally lead friction dominated potentials. After surveying many string vacua and different corners of moduli space he has so far found no cases where such a scenario is possible. 


\section{References}

[1] D. Abbaneo et al. [LEP Electroweak Working Group] and N. de Groot et al. [SLD Heavy Flavor Group], CERN-EP-2002-091 [hep-ex/0212036]; and additional updates at http: / / lepewwg.web.cern.ch/LEPEWWG/.

[2] J.F. Gunion, H.E. Haber, G. Kane and S. Dawson, The Higgs Hunter's Guide (Perseus Publishing, Reading, MA, 1990).

[3] H.E. Haber and Y. Nir, Nucl. Phys. B335, 363 (1990).

[4] J.F. Gunion and H.E. Haber, SCIPP-02-10 and UCD-2002-10 [hep-ph/0207010], Phys. Rev. D67, in press.

[5] H.E. Haber and G.L. Kane, Phys. Rep. 117, 75 (1985).

[6] T. Banks and W. Fischler, arXiv:hep-th/9906038.

[7] P. C. Argyres, S. Dimopoulos and J. March-Russell, Phys. Lett. B 441, 96 (1998) [arXiv:hep-th/9808138].

[8] T. Banks, arXiv:hep-th/0011255.

[9] T. Banks, arXiv:hep-th/0211160.

[10] Critique of Pure String Theory, Talk given at the Strings 2002 Conference, Cambridge, UK, July 2002; A Critique of Pure String Theory: Heterodox Opinions of Diverse Dimensions, manuscript in preparation.

[11] T.Banks, Cosmological Breaking of Supersymmetry, or Little Lambda Goes Back to the Future II, hep-th/0007146; CosMology and QuantuMechanics, Talk given at the Festschrift for Leonard Susskind, Stanford University, May 20-21, 2000; Cosmological M-Theory, SUSY Breaking, and the Cosmological Constant, Talk given at the Strings 2000 Conference, University of Michigan, July 15-20, 2000. Published in the Proceedings.

[12] T. Banks, arXiv:hep-th/0206117.

[13] T. Banks, arXiv:hep-ph/0203066.

[14] T. Banks, "Supersymmetry and Cosmology," talk at Conference, Strings at the Millenium, Pasadena, Jan. 2000

[15] T. Banks and W. Fischler, arXiv:hep-th/0102077.

[16] T. Banks and W. Fischler, arXiv:hep-th/0212113. 
[17] T. Banks, M. Berkooz, S. H. Shenker, G. W. Moore and P. J. Steinhardt, Phys. Rev. D 52, 3548 (1995) [arXiv:hep-th/9503114].

[18] T. Banks, M. Dine, M. Douglas, Phys. Rev. Lett. 88, 131301, (2002), hep$\mathrm{ph} / 0112059$.

[19] A. Anisimov, T. Banks, M. Dine and M. Graesser, Phys. Rev. D 65, 085032 (2002) [arXiv:hep-ph/0106356].

[20] M. Dine, Y. Grossman and S. Thomas, Slepton Flavor Physics at Linear Colliders Contribution to DPF Summer Study on the Future of Particle Physics (Snowmass 2001) hep-ph/0111154.

[21] M. Dine, E. Kramer, Y. Nir and Y. Shadmi, Phys. Rev. D 63, 116005 (2001) [arXiv:hep-ph/0101092].

[22] E. Witten, arXiv:hep-ph/0201018.

[23] M. Dine, Y. Nir and Y. Shadmi, Phys. Rev. D 66, 115001 (2002) [arXiv:hep$\mathrm{ph} / 0206268]$.

[24] See, for example, I. Hinchliffe and N. Kersting, Phys. Rev. D 64, 116007 (2001) [arXiv:hep-ph/0104137].

[25] See for instance, D. Colladay and V.A. Kostelecky, Phys. Rev. D58 116002 (1998), [arXiv:hep-ph/9809521]; R. Bluhm and V. A. Kostelecky, Phys. Rev. Lett. 84, 1381 (2000), [arXiv:hep-ph/9912542]; V. A. Kostelecky and C. D. Lane, Phys. Rev. D 60116010 (1999), [arXiv:hep-ph/9908504].

[26] M. Dine and Y. Shirman, Phys. Rev. D 63, 046005 (2001) [arXiv:hepth/9906246].

[27] T. Banks and M. Dine, Phys.Rev. D45 (1992) 1424, [arXiv:hep-th/9109045].

[28] D.A. MacIntire, [arXiv:hep-th/9308149].

[29] M. Berkooz, R.G. Leigh, J. Polchinski, N. Seiberg and E. Witten, Nucl.Phys. B475 (1996) 115, [arXiv:hep-th/9605184].

[30] E. Witten, Nucl. Phys. B 195, 481 (1982).

[31] M. Fabinger and P. Horava, Nucl.Phys. B580 (2000) 243, [arXiv:hepth/0002073].

[32] M. Dine and A.E. Nelson, [arXiv:hep-ph/9303230], Phys. Rev. D48 (1993) 1277: M. Dine, A. Nelson, Y. Nir and Y. Shirman, Phys. Rev. D53 (1996) 2658, [arXiv:hep-ph/9507378]. 
[33] M. Dine, Phys. Lett. B 482, 213 (2000) [arXiv:hep-th/0002047].

[34] G. Giudice, M.A. Luty, H. Murayama and R. Rattazzi, JHEP 9812 (1998) 027, [arXiv:hep-ph/9810442].

[35] L. Randall and R. Sundrum, Nucl.Phys. B557 (1999) 79, [arXiv:hep-th/9810155].

[36] D.E. Kaplan, G.D. Kribs and M. Schmaltz, Phys.Rev. D62 (2000) 035010, [arXiv:hep-ph/9911293]; Z. Chacko, M.A. Luty, A.E. Nelson and E. Ponton, JHEP 0001 (2000) 003, [arXiv:hep-ph/9911323]; M. Schmaltz and W. Skiba, Phys.Rev. D62 (2000) 095004, [arXiv:hep-ph/0004210].

[37] M. Dine and D.A. MacIntire, Phys. Rev. D46 (1992) 2594, [arXiv:hep$\mathrm{ph} / 9205227]$

[38] A. Anisimov, M. Dine, M. Graesser and S. Thomas, JHEP 0203, 036 (2002) [arXiv:hep-th/0201256].

[39] M. Dine, E. Gorbatov, I. R. Klebanov and M. Krasnitz, arXiv:hep-th/0303076.

[40] A. Sen, JHEP 9808, 012 (1998) [arXiv:hep-th/9805170].

[41] M. Dine, L. Randall and S. Thomas, Nucl. Phys. B 458, 291 (1996) [arXiv:hep$\mathrm{ph} / 9507453]$.

[42] R. Allahverdi, B. A. Campbell and J. R. Ellis, Nucl. Phys. B 579, 355 (2000) [arXiv:hep-ph/0001122].

[43] A. Anisimov and M. Dine, Nucl. Phys. B 619, 729 (2001) [arXiv:hep$\mathrm{ph} / 0008058]$

[44] A. Anisimov, arXiv:hep-ph/0111233.

[45] M. Dine and A. Kusenko, arXiv:hep-ph/0303065.

[46] T. Banks, M. Dine and M. Graesser, arXiv:hep-ph/0210256.

[47] M. Dine, L. Randall and S. Thomas, Supersymmetry Breaking in the Early Universe Phys. Rev. Letters 75 (1995) 398, hep-ph/9503303.

[48] T. Banks, M. Dine, JHEP 0110:012, (2001), hep-th/0106272.

[49] G. Huey, P. J. Steinhardt, B. A. Ovrut and D. Waldram, Phys. Lett. B 476, 379 (2000) [arXiv:hep-th/0001112].

[50] J.F. Donoghue, Spatial Gradients in the Cosmological Constant, hep$\mathrm{ph} / 0101130$. 
[51] M. Dine, arXiv:hep-th/0107259.

[52] J. Garriga and A. Vilenkin, Phys. Rev. D 64, 023517 (2001) [arXiv:hepth/0011262]; J. F. Donoghue, JHEP 0008, 022 (2000) [arXiv:hep-ph/0006088].

[53] N. Arkani-Hamed, H. C. Cheng, P. Creminelli and L. Randall, arXiv:hepth/0301218.

[54] T. Banks, M. Dine and L. Motl, On Anthropic Solutions of the Cosmological Constant Problem, JHEP 0101, 31 (2001) [hep-th/0007206].

[55] R. Bousso and J. Polchinski, JHEP 0006, 006 (2000) [arXiv:hep-th/0004134].

[56] S. Kachru, R. Kallosh, A. Linde and S. P. Trivedi, arXiv:hep-th/0301240.

[57] M. Carena, J.R. Espinosa, M. Quiros and C.E.M. Wagner, Phys. Lett. B355, 209 (1995); M. Carena, M. Quiros and C.E.M. Wagner, Nucl. Phys. B461, 407 (1996); H.E. Haber, R. Hempfling, and A.H. Hoang, Z. Phys. C75, 539 (1997). For a recent update that includes the most complete theoretical analysis, see G. Degrassi, S. Heinemeyer, W. Hollik, P. Slavich and G. Weiglein, hep-ph/0212020.

[58] M. Carena, J.S. Conway, H.E. Haber and J.D. Hobbs et al., FERMILAB-Conf00/270-T [hep-ph/0010338].

[59] ALEPH, DELPHI, L3 and OPAL Collaborations, The LEP working group for Higgs boson searches], LHWG Note 2002-01 (July 2002), contributed paper for ICHEP'02 (Amsterdam, July 2002), and additional updates at http: / / lephiggs . web. cern. ch/LEPHIGGS / www/Wel come.html; P.A. McNamara and S.L. Wu, Rept. Prog. Phys. 65, 465 (2002).

[60] M. Carena, D.W. Gerdes, H.E. Haber, A.S. Turcot and P.M. Zerwas, "Executive summary of the Snowmass 2001 working group (P1): Electroweak Symmetry Breaking," in Proceedings of the APS/DPF/DPB Summer Study on the Future of Particle Physics (Snowmass 2001), edited by R. Davidson and C. Quigg, SNOWMASS-2001-P1001 [hep-ph/0203229].

[61] J.F. Gunion, H.E. Haber and R. Van Kooten, “ Higgs Physics at the Linear Collider," SCIPP-02-37 and UCD-02-18 [hep-ph/0301023], to appear in Linear Collider Physics in the New Millennium, edited by K. Fujii, D. Miller and A. Soni.

[62] M. Carena, H.E. Haber, H.E. Logan and S. Mrenna, Phys. Rev. D65, 055005 (2002) [E: D65, 099902 (2002)].

[63] H.E. Haber, M.J. Herrero, H.E. Logan, S. Penaranda, S. Rigolin and D. Temes, Phys. Rev. D63, 055004 (2001). 
[64] For a review, see M.C. Gonzalez-Garcia and Y. Nir, hep-ph/0202058.

[65] Y. Grossman and H.E. Haber, Phys. Rev. Lett. 78, 3438(1997).

[66] Y. Grossman and H.E. Haber, Phys. Rev. D59, 093008 (1999).

[67] Y. Grossman and H.E. Haber, Phys. Rev. D63, 075011 (2001).

[68] Y. Grossman and H.E. Haber, SLAC-PUB-8173 (1999) [hep-ph/9906310], presented at the DPF-99 Conference.

[69] Y. Grossman and H.E. Haber, Phys. Rev. D67, 036002 (2003).

[70] G. W. Bennett et al. [Muon $g-2$ Collaboration], Phys. Rev. Lett. 89, 101804 (2002) [E: 89, 129903 (2002)].

[71] A. Dedes and H.E. Haber, JHEP 0105, 006 (2001).

[72] D. Chang, W. Chang, C. Chou and W. Keung, Phys. Rev. D63, 091301 (2001); K. Cheung, C. Chou and O.C. Kong, Phys. Rev. D64, 111301 (2001).

[73] F. Larios, G. Tavares-Velasco and C. P. Yuan, Phys. Rev. D64, 055004 (2001).

[74] G. Barenboim, L. Borissov, J. Lykken and A. Yu Smirnov, JHEP0210, 001 (2002) [hep-ph/0108199]; G. Barenboim, L. Borissov and J. Lykken, Phys. Lett. B534, 106 (2002) [hep-ph/0201080]; J. Bachall, V. Barger and D. Marfatia, Phys. Lett. B534, 120 (2002) [hep-ph/0201211]; G. Barenboim, J.F. Beacom, L. Borissov and B. Kayser, Phys. Lett. B537, 227 (2002) [hep-ph/0203261].

[75] M. Graesser and S. Thomas, Phys. Rev. D65, 075012 (2002) [hep-ph/0104254].

[76] S.R. Coleman and F. De Luccia, Phys. Rev. D21, 3305 (1980).

[77] N. Arkani-Hamed, A.G. Cohen and H. Georgi, Deconstructing Dimensions, Phys.Rev.Lett. 86, 4757 (2001) [hep-th/0104005].

[78] A.E. Nelson and M.J. Strassler, Exact Results for Supersymmetric Renormalization and the Supersymmetric Flavor Problem, hep-ph/0104051.

[79] E. Witten, Nucl. Phys. B443, 85 (1995) [arXiv:hep-th/9503124]. 